\title{
1 Intergenerational hormesis is regulated by heritable 18S rRNA methylation
}

2

3 Noa Liberman ${ }^{1,2}$, Maxim V. Gerashchenko ${ }^{3}$, Konstantinos Boulias ${ }^{1,2}$, Fiona G MacWhinnie ${ }^{2}$,

4 Albert Kejun Ying ${ }^{1,2}$, Anya Flood Taylor ${ }^{2}$, Joseph Al Haddad ${ }^{2}$, Hiroki Shibuya ${ }^{1,2,4}$, Lara

5 Roach $^{1,2}$, Anna Dong ${ }^{2}$, Vadim N. Gladyshev ${ }^{3}$, and Eric Lieberman Greer ${ }^{1,2, *}$

8 MA, USA

$9 \quad 2$ Division of Newborn Medicine, Boston Children's Hospital, Boston MA, USA

$10{ }^{3}$ Division of Genetics, Department of Medicine, Brigham \& Women's Hospital, Harvard Medical

11 School, Boston MA 02115, USA

$12{ }^{4}$ Current address: Department of Chemistry and Molecular Biology, University of Gothenburg, 13 SE-40530 Gothenburg, Sweden

14

*Correspondence: Eric L. Greer (eric.greer@childrens.harvard.edu) 
Summary: Heritable non-genetic information can regulate a variety of complex phenotypes. However, what specific non-genetic cues are transmitted from parents to their descendants are poorly understood. Here, we perform metabolic methyl-labelling experiments to track the heritable transmission of methylation from ancestors to their descendants in the nematode Caenorhabditis elegans. We find that methylation is transmitted to descendants in proteins, RNA, DNA and lipids. We further find that in response to parental starvation, fed naïve progeny display reduced fertility, increased heat stress resistance, and extended longevity. This intergenerational hormesis is accompanied by a heritable increase in N6'-dimethyl adenosine $\left(\mathrm{m}^{6,2} \mathrm{~A}\right)$ on the $18 \mathrm{~S}$ ribosomal RNA at adenosines 1735 and 1736 . We identified the conserved DIMT-1 as the $\mathrm{m}^{6,2} \mathrm{~A}$ methyltransferase in C. elegans and find that dimt-1 is required for the intergenerational hormesis phenotypes. This study provides the first labeling and tracking of heritable non-genetic material across generations and demonstrates the importance of rRNA methylation for regulating the heritable response to starvation.

Keywords: transgenerational, intergenerational, heritable methylation, rRNA methylation, $18 \mathrm{~S}$ rRNA, DIMT1, WBSCR22, hormesis, starvation.

Introduction: Organisms have the ability to adapt to different environmental cues and activate stress response pathways to allow for survival under adverse conditions. The ability of an organism to address these conditions is variable, relying not only on their genetic information but also on the non-genetic (epigenetic) information integrated both from their environment and that was transferred to them by their parents. This non-genetic inheritance allows organisms to adapt to extreme environmental conditions and transmit this information to their progeny without mutating the genome. By circumventing mutations, stressed organisms can return to basal conditions once the environment reverts to a more favorable state. While the inheritance of genetic information is well established, inheritance of epigenetic information has been a matter of debate. Even so, growing evidence, both phenotypic and molecular, have greatly supported the biological existence of this concept. Epigenetic inheritance has been shown to regulate physical appearance, energy metabolism, behavioral state and longevity in species ranging from yeast to humans (Boskovic and Rando, 2018; Daxinger and Whitelaw, 2012; Liberman et al., 2019; Lim and Brunet, 2013). More specifically, epigenetic inheritance has been linked to interand transgenerational mechanisms that regulate the response to various environmental cues and stresses (Boskovic and Rando, 2018; Daxinger and Whitelaw, 2012; Lim and Brunet, 2013) including heat stress (Ito et al., 2011; Klosin et al., 2017; Lang-Mladek et al., 2010; Migicovsky et al., 2014; Schott et al., 2014; Seong et al., 2011) and starvation (Demoinet et al., 2017; HouriZeevi et al., 2020; Houri-Zeevi et al., 2019; Jimenez-Chillaron et al., 2009; Jobson et al., 2015; Rechavi et al., 2014; Webster et al., 2018). Food availability is one of the most robust and reproducible environmental cues to induce inter- and transgenerational epigenetic inheritance across a wide variety of species from yeast to mice (Boskovic and Rando, 2018; Liberman et al., 2019; Lim and Brunet, 2013). Correlative evidence has demonstrated that when people experience famine in utero, such as the Dutch Hunger study or the great Chinese famine (Cheng et al., 2020; Li et al., 2010; Lumey et al., 2009; Painter et al., 2008; Pembrey et al., 2006), obesity, diabetes, and cardiovascular diseases can arise. However, what specific non-genetic information is passed from parents to their children to warn the next generation of reduced food availability is still unknown. A large number of studies over the past decade have identified epigenetic phenomena and characterized histone modifying enzymes, prions, small RNA 
pathways, and DNA methylation as necessary components for epigenetic inheritance (Boskovic and Rando, 2018; Daxinger and Whitelaw, 2012; Liberman et al., 2019; Lim and Brunet, 2013). However, while many groups have identified critical epigenetic regulators as being required for epigenetic inheritance, no one has thus far directly demonstrated what is the specific epigenetic information that is transmitted from parents to their progeny.

\section{Results}

\section{Parental Starvation Induces Intergenerational Hormesis in Progeny}

We first set out to establish a system where environmental manipulations caused robust reproducible generational transmission of phenotypes. Parental starvation has been shown to cause altered levels of small RNAs (Houri-Zeevi et al., 2020; Houri-Zeevi et al., 2019; Rechavi et al., 2014), reduce fertility (Demoinet et al., 2017), and increase lifespan, size, and heat stress resistance (Jobson et al., 2015; Rechavi et al., 2014; Webster et al., 2018) in descendants for one or several generations in C. elegans. We therefore wanted to determine whether, in our hands, parental starvation could affect the phenotypes of naïve descendants. Consistent with previous work (Jobson et al., 2015; Rechavi et al., 2014; Webster et al., 2018), we found that seven days of starvation at the first larval stage (L1) of C. elegans development, caused a reduction in fertility, an increase in heat stress resistance, and a subtle extension in lifespan (Figs. 1A-C). Furthermore, parental (P0) starvation caused naïve F1 descendants to also display increased heat stress resistance, reduced fertility, and a subtle extension in lifespan (Figs. 1D-F). These phenotypes persisted in the F2 generation but reverted back to the levels seen in descendants of well-fed worms by the F3 generation (Fig. S1). Thus, parental starvation induced an adaptive response in not only the generation that was exposed to the environmental stress, but also in their naïve descendants. Furthermore, the observation that these phenotypes do not persist in the later generations of naïve descendants, suggests that some epigenetic information is being transferred to regulate these phenotypes.

\section{Heritable Methylation is Elevated in RNA of Descendants of Starved Parents}

To determine what specific epigenetic information is passed from parents to their progeny, we first designed and optimized a system for tracking inherited non-genetic material. We decided to focus on methylation, due to the versatility of substrates and their use of this small chemical moiety to alter their function and to respond to the environment. Methionine is used to generate S-adenosylmethionine (SAM) which is the predominant methyl donor for DNA, RNA, lipids, and proteins (Champe and Harvey, 1994). We therefore used modified SAM where the hydrogens of the methyl group are replaced with the heavy isotope deuterium (D) or the radioactive isotope tritium $\left({ }^{3} \mathrm{H}\right)$ to allow us to detect and track methylation. Modified SAM and methionine have previously been used to detect direct methylation targets (Boulias et al., 2019; Islam et al., 2011; Mann and Smith, 1977; Wang et al., 2011). To ensure that modified SAM could be used efficiently by methyltransferases, we performed in vitro methylation assays with SAM-D ${ }_{3}$ and $\mathrm{SAM}_{-}{ }^{3} \mathrm{H}_{3}$ (Fig. S2). SAM- $-{ }^{3} \mathrm{H}_{3}$ was utilized by the DNA C5-cytosine methyltransferase HpaII (Mann and Smith, 1977) as detected by scintillation counting of methylated substrates (Fig. S2A). In addition, we had previously demonstrated that $\mathrm{SAM}-{ }^{3} \mathrm{H}_{3}$ could also be utilized by the RNA N6, 2'-O-dimethyladenosine $\left(\mathrm{m}^{6} \mathrm{Am}\right)$ methyltransferase PCIF1 and by the histone methyltransferases SET-2, SET-17, SET-26, and SET-30 (Boulias et al., 2019; Greer et al., 2014; Greer et al., 2010). Similarly, we found that SAM-D 3 was an efficient methyl donor for both DNA and RNA methyltransferases by performing in vitro 
methylation assays with the rRNA methyltransferase METL-5 and the DNA adenine methylase, dam (Nikolskaya et al., 1981), and performing ultra-high performance liquid chromatography coupled with mass spectrometry to detect the modified nucleosides (UHPLC-ms/ms) (Fig. S2B and (Liberman et al., 2020)).

To determine whether methylation is transmitted from parents to progeny and to examine which substrates were heritably methylated, we administered SAM- ${ }^{3} \mathrm{H}_{3}$ to wildtype early larval stage 4 (L4) C. elegans and then tested for the incorporation of tritium in the total lysate as well as purified DNA, lipids and RNA of adult worms and their progeny. Since the modified methyl label is fed only in the parental generation, any detected tritium in the descendant generation must represent heritable methylation. Indeed, we found that we could detect methylated material in the P0 worms and in naïve F1 eggs. Tritiated methylation was detected in proteins, DNA, lipids, and RNA (Fig. 2A). As far as we are aware, this is the first tracking of epigenetic material from ancestors to their descendants. Since each worm has 250 progeny, it is not feasible, at this stage, to track heritable methylation to the F2 generation since the signal is quickly diluted. It has been shown that SAM is relatively unstable (Morana et al., 2002; Parks and Schlenk, 1958) and therefore it is most likely that any tritium detected in the progeny would have been incorporated into heritably methylated material in the parents and transmitted to the progeny rather than taken up by the progeny themselves or transmitted in the form of SAM- ${ }^{3} \mathrm{H}_{3}$ to be used by the progeny themselves. To further eliminate the possibility of the progeny taking up new $\mathrm{SAM}-{ }^{3} \mathrm{H}_{3}$ we examined methylation levels in F1 eggs which do not consume any nutrients themselves.

The recent growing evidence for RNA's role in transgenerational inheritance led us to initially focus our study on heritable RNA methylation. Other heritably methylated substrates will be interesting to follow up on in independent subsequent studies. Therefore, our next step was to determine whether starvation would affect the amount of heritable methyl moieties on the RNA. We kept arrested L1 worms in the absence of food for 7 days, followed by recovery of the worms on food until they reached the L4 stage. At this point we supplemented the worms with SAM $-{ }^{3} \mathrm{H}_{3}$ and let them continue developing to become egg bearing adults. F1 eggs were extracted, RNA purified and incorporation of radioactivity was measured by scintillation counting (Fig. 2B). We found that there was an increase in detection of radioactivity in RNA of starved parents and their naïve progeny (Fig. S2C). Similarly, we found that there was an increase in radioactive methyl groups in the parental and the naïve progeny generation when ${ }^{3} \mathrm{H}$ methionine was fed to the starved P0 at the L4 stage (Fig. 2C). Worms that were starved did not consume more food once they reached the L4 stage, after recovery on food, as assessed by consumption of bacteria expressing GFP (Fig. S2D), suggesting that there is not an increase in consumption of the methyl donor. Furthermore, we did not detect an increase in heritable methylation in response to parental heat stress (Fig. S3A and B), an environmental cue that has also been shown to elicit transgenerational effects in C. elegans (Klosin et al., 2017; Schott et al., 2014), suggesting that this increase in heritable RNA methylation is a specific response to parental starvation.

\section{Heritable Dimethylation at the N6 Position of Adenines is Elevated on the 18S Ribosomal RNA of Descendants of Starved Parents}

To examine in an independent manner whether and where methylation increased in RNA of descendants whose parents were starved, we repeated the starvation assay feeding Methionine- $\mathrm{D}_{3}$ to the parents (Fig. 2B). Since ribosomal RNA (rRNA) constitutes $>80 \%$ of the total RNA in a cell (Blobel and Potter, 1967), we hypothesized that any observable change in 
heritable methylation on total RNA would occur on rRNA. To determine which rRNAs displayed increased heritable methylation, we isolated total RNA from F1 eggs, whose parents were either fed or starved and were supplemented with Methionine- $\mathrm{D}_{3}$. We then separated the RNA on a gel to isolate $26 \mathrm{~S}, 18 \mathrm{~S}$, and $5.8 / 5 \mathrm{~S}$ rRNAs. To identify which specific methylation modification changes in response to parental starvation, we performed UHPLC-ms $/ \mathrm{ms}$ on each population of rRNA. We found a consistent increase in dimethylated $\mathrm{N} 6$ adenosine $\left(\mathrm{m}^{6,2} \mathrm{~A}\right)$ on the $18 \mathrm{~S}$ rRNA in response to parental starvation (Fig. 2D). We did not observe a consistent increase in methylation of other residues on the $18 \mathrm{~S}$ or on the $26 \mathrm{~S}$ rRNA (Fig. 2D). Together, this data suggests that starvation causes parents to transmit increased $\mathrm{m}^{6,2} \mathrm{~A}$ methylated $18 \mathrm{~S}$ rRNA to their naïve descendants.

\section{Knock-down of dimt-1 decreases 18S rRNA N6-dimethyladenosine and deletion of bud-23 decreases 18S rRNA N7-methylguanosine}

To determine whether $\mathrm{m}^{6,2} \mathrm{~A}$ is important for the intergenerational hormesis phenotypes we observed (Fig. 1), we first sought to identify the enzyme responsible for N6-dimethylation of the $18 \mathrm{~S}$ rRNA in $C$. elegans. The $18 \mathrm{~S}$ has been found to be N6-dimethylated at two adjacent adenosines, 1850 and 1851, in mammals (Sergiev et al., 2018) which correspond to adenosine 1735 and 1736 in the $C$. elegans $18 \mathrm{~S}$ rRNA. These two adjacent 18S rRNA adenosines display conserved methylation from bacteria to humans, and have been shown to be methylated by dimethyladenosine transferase 1 (DIMT1) in humans (Lafontaine et al., 1994; Shen et al., 2020; Suvorov et al., 1988). Another nucleoside on the $H$. sapiens $18 \mathrm{~S}$ rRNA which is physically quite close to the adjacent methylated adenosines and has been shown to be methylated in yeast and humans (Sergiev et al., 2018), is guanosine 1639 which corresponds to guanosine 1531 in the $C$. elegans $18 \mathrm{~S}$ rRNA. The putative N7-guanosine methyltransferase Bud23 in yeast and WBSCR22 in humans has been proposed to methylate this guanosine (Haag et al., 2015; White et al., 2008; Zorbas et al., 2015). Both DIMT1 and Bud23 have been shown to be important for ribosomal RNA processing (Zorbas et al., 2015). Both of these enzymes have clear homologs in C. elegans; E02H1.1 shows homology to DIMT1, and we therefore renamed this gene dimt-1, while C27F2.4 shows homology to Bud23/WBSCR22 (Zhu et al., 2018), which we have therefore renamed bud-23. We wanted to test whether DIMT-1 and BUD-23 were $\mathrm{m}^{6,2} \mathrm{~A}$ and $\mathrm{m}^{7} \mathrm{G} 18 \mathrm{~S}$ rRNA methyltransferases in C. elegans, respectively. We knocked down dimt-1 and bud-23 by feeding wildtype worms bacteria expressing an empty vector (EV) or double stranded RNA against each of these genes. We next extracted total RNA, separated 26S, $18 \mathrm{~S}$, and 5.8S/5S rRNAs, and performed UHPLC-MS/MS on each population of rRNA. We found no discernable changes in rRNA methylation on the $26 \mathrm{~S}$ or $5.8 \mathrm{~S} / 5 \mathrm{~S}$ rRNAs in response to dimt-1 or bud-23 knock-down. However, we did detect a significant decrease specifically in $\mathrm{m}^{6,2} \mathrm{~A} 18 \mathrm{~S}$ rRNA levels without changes in other methylated bases in response to dimt-1 knock-down (Figs. 3A and S4A). Additionally, knocking down bud-23 caused a significant decrease specifically in $\mathrm{m}^{6,2} \mathrm{~A}$ and $\mathrm{m}^{7} \mathrm{G} 18 \mathrm{~S}$ rRNA methylation without affecting other methylated bases (Figs. 3A and S4A). To test whether the change in $18 \mathrm{~S}$ rRNA methylation was due to bud-23 knockdown rather than an off-target effect of the small interfering RNA, we examined RNA methylation in a genetic mutant strain bud-23(tm5768) which contains a large deletion of the putative methyltransferase domain (Zhu et al., 2018). This mutant strain displayed a complete elimination of $18 \mathrm{~S} \mathrm{rRNA} \mathrm{m}^{7} \mathrm{G}$, a substantial decrease in $18 \mathrm{~S}$ rRNA $\mathrm{m}^{6,2} \mathrm{~A}$, and, interestingly, a near doubling of $18 \mathrm{~S}$ rRNA $\mathrm{m}^{6} \mathrm{~A}$ without changing other methylations on the $18 \mathrm{~S}$ rRNA (Figs. 3B and S4B). To determine whether the change in $\mathrm{m}^{7} \mathrm{G}$ was due to BUD-23 activity, we generated transgenic 
rescue strains of WT or G63E/D82K double mutant bud-23 driven by the ubiquitous eft-3 promoter in a bud-23(tm5768) mutant background $\left(P_{\text {eft }-3:}: b u d-23 \mathrm{WT}\right.$ and $P_{\text {eft }-3}:: b u d-23$ G63E/D82K). Equivalent amino acids substitutions have been shown to eliminate WBSCR22 activity in HEK293 cells (Haag et al., 2015). Six independent $P_{\text {eft-3 }}:$ bud-23 WT but not six independent $P_{\text {eft-3: }}:$ bud-23 G63E/D82K lines rescued the $18 \mathrm{~S}$ rRNA m ${ }^{7} \mathrm{G}$ levels (Fig. 3C). These results indicate that BUD-23 catalytic activity is required for $18 \mathrm{~S}$ rRNA methylation. Together these results suggest that DIMT-1 regulates 18S rRNA dimethylation on the N6 position of adenines while BUD-23 regulates both 18S rRNA dimethylation on the N6 position of adenines and methylation on the N7 position of guanines. Since bud-23 knockdown and knock-out caused a decrease in not only $\mathrm{m}^{7} \mathrm{G}$ but also $\mathrm{m}^{6,2} \mathrm{~A}$ levels, this suggests that either BUD-23 can methylate both guanine and adenine or that $\mathrm{m}^{7} \mathrm{G}$ methylation proceeds and facilitates $\mathrm{m}^{6,2} \mathrm{~A}$ methylation. $P_{\text {eft-3: }}:$ bud-23 WT also rescued the $18 \mathrm{~S} \mathrm{~m}^{6,2} \mathrm{~A}$ levels (data not shown) reinforcing the model that $\mathrm{m}^{7} \mathrm{G}$ methylation proceeds and facilitates $\mathrm{m}^{6,2} \mathrm{~A}$ methylation. It was interesting to observe that there was not only a decrease in $\mathrm{m}^{6,2} \mathrm{~A}$ but this was accompanied by an increase in $18 \mathrm{~S}$ rRNA $\mathrm{m}^{6} \mathrm{~A}$ in response to deletion of $b u d-23$. We had previously demonstrated that there is a single adenine on the $C$. elegans $18 \mathrm{~S}$ rRNA that is constitutively methylated by metl-5/METTL5 (Liberman et al., 2020). Therefore, this finding raises the possibility that $\mathrm{m}^{7} \mathrm{G}$ methylation is a necessary precursor for $\mathrm{m}^{6,2} \mathrm{~A}$ methylation, and without $\mathrm{m}^{7} \mathrm{G}$ methylation the adenines which would normally be dimethylated to produce $\mathrm{m}^{6,2} \mathrm{~A}$ are instead singly methylated to produce $\mathrm{m}^{6} \mathrm{~A}$. We could not examine dimt-1 mutant strains as this gene is essential for viability.

To determine whether adenosines 1735 and 1736 and guanosine 1531 are the nucleosides that are $\mathrm{m}^{6,2} \mathrm{~A}$ and $\mathrm{m}^{7} \mathrm{G}$ methylated on the $18 \mathrm{~S}$ rRNA in $C$. elegans, we performed site-specific cleavage and radioactive-labeling followed by ligation-assisted extraction and thin-layer chromatography (SCARLET) (Liu et al., 2013) on 18S rRNA purified from WT and bud23(tm5768) mutant worms. We identified that guanosine 1531 is a conserved N7-methylated nucleoside in C. elegans, and that this residue is N7-methylated constitutively in WT C. elegans (Fig. 3D). The bud-23(tm5768) mutant worms 18S rRNA were almost completely unmethylated at guanosine 1531 (Fig. 3D), suggesting that BUD-23 is responsible for N7-methylation of this specific guanosine. We further found that adenosines 1735 and 1736 were dimethylated on the N6 position (Fig. 3e). Interestingly in the bud-23(tm5768) mutant worms, adenosine 1735 and 1736 displayed reduced dimethylation and increased monomethylation on the N6 position (Fig. $3 \mathrm{E})$. Because $\mathrm{m}^{6,2} \mathrm{~A}$ interferes with Watson-Crick base pairing, the SCARLET method cannot accurately quantify the percentage methylation that is occurring at these residues (personal communication Tao Pan). However, we can conclude that adenosines 1735 and 1736, as well as the human $18 \mathrm{~S}$ adenosine 1850 , are not constitutively $\mathrm{m}^{6,2} \mathrm{~A}$ and therefore these residues are poised to respond to environmental conditions. Interestingly, a recent report also found that in $S$. cerevisiae and mammalian cell lines these two residues can be N6-monomethylated and that $\mathrm{m}^{6} \mathrm{~A}$ increases in response to sulfur starvation (Liu et al., 2021). Thus, our results validate that adenosine 1735 and 1736 are conserved N6-dimethylated residues and that guanosine 1531 is a conserved N7-methylated residue in C. elegans. Furthermore, bud-23 deletion limits N6dimethylation of adenosine 1735 and 1736 and facilitates the N6 monomethylation of these precise adenosines.

\section{DIMT-1 dimethylates 18S rRNA on the N6 position of adenosines 1735 and 1736}

To determine whether DIMT-1 directly methylates $18 \mathrm{~S}$ rRNA, we expressed a glutathione S-transferase (GST)-tagged dimt-1 in bacteria, purified DIMT-1 (Fig. 3F), and 
analyzed its ability to methylate $18 \mathrm{~S}$ rRNA from bud-23 mutant worms, which have reduced levels of $\mathrm{m}^{6,2} \mathrm{~A}$. Recombinant DIMT-1 specifically caused an increase in $\mathrm{m}^{6,2} \mathrm{~A}$ methylation on $18 \mathrm{~S}$ rRNA in vitro (Fig. 3G). To further verify that DIMT-1 is an $18 \mathrm{~S}$ rRNA methylase, we mutated the conserved glutamic acid E79, as the equivalent amino acid is essential for human DIMT-1's catalytic activity (Shen et al., 2020). Mutation of glutamic acid 79 to alanine (E79A) ablated the N6-adenosine dimethyltransferase activity on 18S rRNA from bud-23 mutant worms (Fig. 3G). To determine whether DIMT-1 could methylate adenines 1735 and 1736 within the $18 \mathrm{~S}$ sequence, we performed in vitro methylation assays with recombinant DIMT-1 using 23nucleotide synthetic oligonucleosides consisting of adenosines 1735 and 1736 and flanking nucleosides from the 18S rRNA sequence (Fig. 3H). We found that WT DIMT-1, but not catalytically dead DIMT-1, methylated this oligonucleoside (Fig. 3H). This methylation was specific to adenosines 1735 and 1736, as there was no methylation detected in in vitro methylation assays using the same oligos where adenosines 1735 and 1736 had been replaced by guanosines (Fig. $3 \mathrm{H}$ ). Together these results show that DIMT-1 is the direct $18 \mathrm{~S}$ rRNA m ${ }^{6,2} \mathrm{~A}$ methyltransferase both in vitro and in vivo.

\section{Ribosome profiling reveals bud-23 and dimt-1 knock-down alters translation of genes involved in longevity and the stress response as does parental starvation}

We and others, have found that rRNA methylation alters the association of the ribosome to particular transcripts (Basu et al., 2011; Liberman et al., 2020; Schosserer et al., 2015). To determine what is the consequence of starvation induced heritable rRNA methylation on translation, we first examined the polysome profile of F1 fed and F1 starved wildtype worms. We found that there was no change in polysome profiles in descendants whose parents had been fed or starved (Fig. S5). Thus, parental starvation does not globally affect polysome profiles in their descendants. To determine whether parental starvation alters ribosome binding or association levels of specific transcripts, ribosome-bound RNAs and total cellular polyadenylated-selected RNA were sequenced (Ingolia et al., 2009) in six independent biological replicates from F1 eggs of WT fed or starved parents and four independent biological replicates from eggs of empty vector (EV) control or bud-23 and dimt-1 knockdown worms. We first analyzed the transcription changes in response to bud-23 and dimt-1 knockdown. We found that there was a high degree of overlap between genes which were misregulated after bud-23 knockdown and those which were misregulated after dimt-1 knockdown (Figs. 4A and B, Supplementary Table 2, 1224 of the 1319 upregulated genes upon bud-23 knockdown were upregulated after dimt-1 knockdown and 731 of the 882 downregulated genes upon bud-23 knockdown were downregulated after dimt 1 knockdown $\mathrm{p}=0$ by hypergeometric probability). A gene ontology (GO) analysis of the shared misregulated genes in response to bud-23 and dimt-1 knockdown revealed genes involved in reproduction, translation, longevity, and growth (Fig. S6B). Parental starvation also caused a change of gene expression enriched in the response to heat, translation, and the endoplasmic reticulum unfolded protein response (Figs. 4C, S6C, and Supplementary Table 2). Parental starvation had no effect on the expression levels of bud-23 or dimt- 1 themselves (Fig. S6D), suggesting that if bud-23 and dimt-1 are necessary for the intergenerational hormesis phenotypes it is due to inheritance of methylated rRNAs rather than altered inheritance of the methyltransferases themselves. Despite the fact that EV, bud-23, and dimt-1 knockdown worms were fed HT115(DE3) bacteria and the F1 WT fed and F1 WT starved were fed OP50-1 bacteria, there was still a significant overlap between genes which were downregulated upon knockdown of bud-23 and dimt-1 and those which were upregulated in 
response to parental starvation (Fig. 4D, 195 of the 731 shared downregulated genes in response to bud-23 and dimt-1 knockdown were upregulated in response to parental starvation $\mathrm{p}<7 \mathrm{E}-27$ by hypergeometric probability). Interestingly, the shared pathways which become transcriptionally dysregulated in response to knock-down of the $18 \mathrm{~S}$ rRNA methyltransferases and parental starvation include translation, the response to heat, development, and reproduction (Fig. 4E), which mirror some of the phenotypic responses we observe in response to parental starvation (Fig. 1). Examining the shared transcriptionally dysregulated pathways in response to knockdown of the $18 \mathrm{~S}$ rRNA methyltransferases and parental starvation in an alternative annotation methodology, WormCat (Holdorf et al., 2020), also revealed dysregulation of genes involved in stress responses and the ribosome (Fig. S6E). This suggests that a portion of these intergenerational phenotypes might be due to transcriptional dysregulation.

To determine if parental starvation and bud-23 and dimt-1 knockdown also altered the ribosome binding or association levels of specific transcripts, we sequenced ribosome-bound RNAs from the same six and four biological replicates and normalized that to the levels of the transcripts to measure translation efficiency. We observed a high degree of reproducibility within our replicate samples (Fig. S7A and Supplementary Table 3). Similarly, to the shared transcriptional response to knock-down of bud-23 and dimt-1, there was also a similar translational response as assessed by translation efficiency (Figs. 4F and S7B). We found that 1103 transcripts were differentially bound after dimt-1 knockdown and 62 transcripts were differentially bound after $b u d-23$ knockdown. While we only detected 62 differentially bound transcripts after bud-23 knockdown that met our rigorous statistical standards, 52 of these genes were also differentially bound by the ribosome after dimt -1 knockdown ( $p<1.6 \mathrm{E}-46$ by hypergeometric probability). Additionally, a gene ontology analysis of genes which were differentially bound after dimt-1 and bud-23 knockdown revealed similar pathways were alternatively bound including determination of adult lifespan, development, and reproduction (Figs. 4G, S7C, and S7D). There was also a difference between transcripts bound by the ribosome after parental starvation compared to progeny whose parents had been well-fed (Figs. $4 \mathrm{H}$ and S7E). Interestingly, the genes which were differentially translated by the ribosome in response to parental starvation displayed a high degree of overlap with the transcripts which were differentially translated in response to dimt-1 KD (Fig. S7F, 76 of 443 starvation alternatively bound transcripts were differentially bound after dimt- 1 knockdown, $\mathrm{p}<1 \mathrm{E}-9$ by hypergeometric probability). The shared transcripts which were differentially translated by the ribosome in response to parental starvation or dimt-1 knockdown were those that are involved in the altered phenotypes observed after parental starvation, including longevity, reproduction and the cellular response to stress (Figs. 4I and S7G). Together these results suggest that parental starvation causes both transcriptional and translational changes that could explain the observed phenotypic changes and that $18 \mathrm{~S}$ rRNA methylation by the $\mathrm{m}^{6,2} \mathrm{~A}$ and $\mathrm{m}^{7} \mathrm{G}$ methyltransferases could be responsible for the intergenerational phenotypic changes. This raises the possibility that parental starvation can transmit specific modified rRNAs to their naïve descendants to prime the descendant worms for a possible starvation.

\section{DIMT-1 and BUD-23 are Required for Intergenerational Hormesis in Response to Starvation}

To determine whether the $\mathrm{m}^{6,2} \mathrm{~A}$ modification is important for the intergenerational hormesis phenotypes we observed (Fig. 1), we examined whether knock-down of bud-23 or dimt- 1 would eliminate the intergenerational hormesis phenotypes. We found that knocking 
down bud-23 or dimt-1 had no effect in the parental generation response to starvation, since starvation of $\mathrm{P} 0$ bud-23 or dimt-1 knockdown worms reduced fertility and increased heat stress resistance (Figs. 5A, B). Excitingly, however, knock-down of both bud-23 and dimt-1 completely eliminated the increased heat stress resistance and reduction in fertility in the naïve $\mathrm{F} 1$ generation progeny whose parents had been starved relative to the $\mathrm{F} 1$ generation progeny whose parents had been fed (Figs. 5C, D). Knock-down of bud-23 and dimt-1 did cause a reduction in fertility and increase in heat stress resistance in the fed progeny relative to empty vector fed control progeny, suggesting that, independent of parental starvation, $18 \mathrm{~S} \mathrm{rRNA} \mathrm{m}{ }^{6,2} \mathrm{~A}$ and $\mathrm{m}^{7} \mathrm{G}$ methylation is important for fertility and stress resistance. Despite the starvationindependent consequence of bud-23 and dimt-1 knock-down in F1 generation progeny, there was still the possibility of a further reduction in fertility or increase in heat stress resistance which did not occur, suggesting that BUD-23 and DIMT-1 are necessary for the transmission of intergenerational hormesis. C. elegans consume the E. coli strain OP50-1 as their standard diet, but for feeding double-stranded RNA to C. elegans, to knock-down a gene, we use an E. coli strain, HT115, which lacks RNAase III, and therefore does not degrade the double stranded RNA that is produced in the bacteria (Timmons et al., 2001). While the switch from OP50-1 to HT115 bacteria, did not affect the heat stress resistance and fertility phenotypes, starved worms placed on HT115 bacteria did not display the subtle increase in lifespan that starved worms display on OP50-1 (Fig. S8A). Therefore, we could not determine whether DIMT-1 was necessary for the intergenerational lifespan extension in response to parental starvation. We did find that bud23(tm5768) mutant worms displayed no increase in lifespan in the parental generation in response to starvation (Fig. S8B), suggesting that BUD-23 is required for the extension in lifespan in response to starvation. Therefore, we could not examine whether BUD-23 was necessary for the intergenerational lifespan extension in response to parental starvation. Similarly to the knock-down phenotypes, the $b u d-23$ genetic mutant, $t m 5768$, while generally more stress resistant and less fertile than WT worms, still displayed an increase in heat stress resistance and a reduction in fertility in response to starvation in the parental generation (Figs. 5E, F) but the naïve bud-23(tm5768) F1 progeny failed to display an increase in heat stress resistance and reduction in fertility relative to bud-23(tm5768) F1 progeny from fed parents that WT worms displayed (Figs. 5G, H). Because bud-23(tm5768) mutant worms could still display a significant change in fertility and heat stress response after starvation in the P0 generation (Figs. 5E, F), these results suggest that both heritable starvation dependent and independent phenotypes can be assessed. Thus, these results suggest that BUD-23 and DIMT-1 are necessary for the transmission of the intergenerational hormesis response to starvation. Collectively, these data suggest that in response to starvation, worms transmit elevated N6 dimethylated adenosine 18S rRNA to their naïve progeny, which helps to confer an intergenerational hormesis phenotype.

\section{Discussion:}

Thus far, correlations have been reported of altered histone modifications, DNA methylation, or small RNA levels in naïve descendants which display transgenerational epigenetic inheritance phenotypes (reviewed in (Boskovic and Rando, 2018; Daxinger and Whitelaw, 2012; Liberman et al., 2019; Lim and Brunet, 2013)). Several groups have elegantly demonstrated how histone modifying enzymes or small RNA machinery are required for transgenerational epigenetic inheritance phenotypes (Boskovic and Rando, 2018; Daxinger and Whitelaw, 2012; Gaydos et al., 2014; Kaletsky et al., 2020; Liberman et al., 2019; Lim and Brunet, 2013). However, no one has yet tracked non-genetic material across generations. Non- 
genetic material could include proteins, non-coding RNA, chromatin modifications, or any chemical modification to the transcriptome or proteome. As far as we are aware, this is the first report directly tracking non-genetic information from parents to their progeny. We have identified that parents, in response to starvation, transmit increased $\mathrm{m}^{6,2} \mathrm{~A}$ methylated $18 \mathrm{~S}$ rRNA to their naïve progeny (Fig. 2). We further identified that DIMT-1 is the methyltransferase required for N6-dimethylating adenosine 1735 and 1736 on the 18S rRNA and that BUD-23 is the putative N7-methyltransferase for guanosine 1531 (Fig. 3). We found that these methylations and parental starvation affect the ribosome occupancy of the F1 generation at transcripts involved in longevity regulation, stress response, and reproduction (Figs. 4, S6 and S7). Excitingly, we found that BUD-23 and DIMT-1 are required for the intergenerational hormesis phenotypes of reduced fertility and increased heat stress in response to parental starvation (Fig. 5). Together, these data track heritable methylation across a generation and identify methylated ribosomal RNA as a necessary career of non-genetic information in response to starvation.

What could be the advantage of transmitting premethylated 18S rRNA to progeny of starved parents? Since $\mathrm{m}^{6,2} \mathrm{~A}$ on adenosines 1850 and 1851 and $\mathrm{m}^{7} \mathrm{G}$ on guanosine 1639 in mammals, and the corresponding nucleosides in yeast, have primarily been implicated in rRNA processing (Haag et al., 2015; Letoquart et al., 2014; Shen et al., 2020; White et al., 2008; Zorbas et al., 2015). Our examination of worm rRNA methylation at these residues suggests that methylation at guanosine 1531 is virtually constitutive while methylation at adenosines 1735 and 1736 are variable. Therefore, it is possible that the starved nematodes are passing along more processed rRNAs to their progeny than their fed counterparts. To support this notion, it was recently demonstrated that maternally provided ribosomes are sufficient to allow the worms to proceed to the L1 stage (Cenik et al., 2019). Skipping the methylation processing steps of rRNAs might give the progeny a slight advantage in rapidly translating proteins while still dependent on the maternal ribosomes for translation. Alternatively, the difference in amounts of $^{6,2} \mathrm{~A}$ methylated or unmethylated rRNAs in the progeny could cause alterations in ribosome heterogeneity which would potentially facilitate the translation of specific stress response and reproduction genes. It will be exciting in future experiments to identify whether one or both of these theories is in play here.

The dual adjacent N6-dimethylated adenosines on the 18S rRNA are in a helix which is physically adjacent to the peptidyl site (P-site), which will hold the tRNA as it is linked to the growing polypeptide chain during translation (Polikanov et al., 2015; Sergiev et al., 2018). Crystal structure analysis has revealed that methylation of these adenosine residues in $T$. thermophilus facilitates appropriate packing of the rRNA, and that absence of these methylations disrupts the rRNA structure in the A and P sites of the ribosome (Demirci et al., 2010). These residues are also directly in contact with a ribosomal protein that bridges the large subunit and the small subunit of the ribosome, potentially explaining why these methylation events alter translation efficiency (Sloan et al., 2017). Lack of N6-dimethylated adenosines on the 18S rRNA has been shown to decrease fidelity during elongation (van Buul et al., 1984) and to increase translation from non-AUG codons (O'Connor et al., 1997). These subtle changes in rRNA structure could therefore explain the altered translation profile we observe in response to starvation and knock-down of dimt-1 and bud-23 (Fig. 4). It will be interesting, in future experiments, to determine whether there is some unique common property associated with the differentially bound transcripts in response to parental starvation and knock-down of dimt-1 and 
bud-23 and whether $\mathrm{m}^{6,2} \mathrm{~A}$ on adenosines 1735 and 1736 alters the ribosomes capacity to bind to transcripts involved in reproduction, heat stress resistance, and longevity.

We identified several molecules in addition to rRNA that could be heritably methylated (Fig. 2A). Since there is only partial overlap between mis-regulated gene expression and translation in response to bud-23 and dimt-1 knock-down and parental starvation (Fig. 4), it is probable that other heritable epigenetic information is important for controlling the descendant response to parental starvation. It will be intriguing, in future studies, to examine these methylated molecules, including other types of RNA, proteins, and lipids, and their possible participation in the transfer of information from parents to progeny. It will also be interesting to explore if they respond to environmental stimuli and if they are important for the organism's ability to appropriately respond to extreme environmental cues that their parents or grandparents experienced. Due to the dilution of these metabolic methyl labels after a single generation, it is currently not feasible to examine whether these molecules could be transgenerationally transmitted, however, it will be exciting in subsequent studies to determine what non-genetic information can persist for multiple generations, or whether and how a non-genetic cue could be reacquired for a specific number of generations.

Acknowledgments: We are grateful to S. Guang and T.K. Blackwell for strains and reagents, and to E. Pollina and M. Greenberg for sequencing ribosome sequencing libraries, and T. Pan for advice about SCARLET. We thank D. Moazed, A. Brunet, and members of the Greer laboratory for discussions and feedback on the manuscript. This work was supported by NIH grants (R00AG043550, DP2AG055947) to E.L.G..

Author contributions: E.L.G conceived the study and wrote the paper. N.L. and E.LG. planned the study. N.L. developed and optimized UHPLC-ms/ms methods and metabolic methyl labelling experiments, produced Figs. 2, S2 and S3, and generated samples for ribosome and mRNA sequencing, and advised F.G.M., A.F.T. and A.D.. M.V.G. performed ribosome sequencing and mRNA sequencing experiments and subsequent analysis for Figs. 4, S5 and S6. K.B. produced Figs. 3D and $3 \mathrm{E}$ and generated transgenic rescue worms and advised A.K.Y. and J.A.H. F.G.M. helped generate Figs. 2A and S2. A.K.Y. performed initial UHPLC-ms $/ \mathrm{ms}$ methods and was advised by K.B.. A.F.T. performed initial phenotypic characterization experiments. J.A.H. produced Fig. 3C. H.S. and L.R. performed initial metabolic methyl labeling experiments. A.D. helped optimize phenotypic characterization experiments. V.N.G. advised M.V.G.. E.L.G. produced Figs. 1, 3A, 3B, 3F, 3G, 3H, 5, S1, S4, S6, S7, and S8. All authors discussed the results and commented on the manuscript.

Declaration of interests: Authors declare no competing interests.

Contact for Reagent and Resource Sharing: Please contact E.L.G. (eric.greer@childrens.harvard.edu) for reagents and resources generated in this study.

Data and Software Availability: Raw sequencing data can be accessed through the GEO repository. 


\section{Figure legends}

480 Fig. 1 Parental starvation causes intergenerational hormesis in descendants

481

482

483

484

485

486

487

488

489

490

491

492

493

494

495

496

497

498

499

500

501

502

503

504

505

506

507

508

509

510

511

512

513

514

515

516

517

518

519

520

521

522

523

524
A, Starvation causes a reduction in reproduction. Each column represents the mean $\pm \mathrm{SEM}$ of 4 independent experiments performed in three plates with 10 worms per plate. Dots are color coded to display matched independent experiments. ${ }^{* *} \mathrm{p}<0.01$ as assessed by paired $\mathrm{t}$ test. $\mathbf{B}$, Starvation causes an increase in survival in response to $37^{\circ} \mathrm{C}$ heat stress for 6 hours. Each column represents the mean \pm SEM of 4 independent experiments performed in three plates with 30 worms per plate. Dots are color coded to display matched independent experiments. ${ }^{* *} \mathrm{p}<0.01$ as assessed by paired t test. $\mathbf{C}$, Starvation causes a subtle increase in longevity. Each condition represents three plates of $\sim 30$ worms per plate. This is a representative experiment which has been performed 5 times. Statistics of independent experiments are presented in Supplementary Table 1. ${ }^{* * *} \mathrm{p}<0.001$ as assessed by Log-rank (Mantel-Cox) test. D, Naïve F1 progeny whose parents were starved have reduced fertility relative to progeny whose parents were fed. Each column represents the mean \pm SEM of 4 independent experiments performed in three plates with 10 worms per plate. Dots are color coded to display matched independent experiments. ${ }^{*} \mathrm{p}<0.05$ as assessed by paired t test. E, Naïve F1 progeny whose parents were starved display an increase in survival in response to $37^{\circ} \mathrm{C}$ heat stress for 6 hours. Each column represents the mean $\pm \mathrm{SEM}$ of 6 independent experiments performed in three plates with 30 worms per plate. Dots are color coded to display matched independent experiments. ${ }^{* *} \mathrm{p}<0.01$ as assessed by paired $\mathrm{t}$ test. $\mathbf{F}$, Naïve F1 progeny whose parents were starved have a subtle increase in longevity. Each condition represents three plates of $\sim 30$ worms per plate. This is a representative experiment which has been performed 5 times. Statistics of independent experiments are presented in Supplementary Table $1 .{ }^{* *} \mathrm{p}<0.01$ as assessed by Log-rank (Mantel-Cox) test.

\section{Fig. 2 Descendants of starved parents display increased $\mathrm{m}^{6,2} \mathrm{~A} 18 \mathrm{~S}$ rRA methylation} A, Radioactive methyl groups were detected in the total lysate, DNA, lipids, or RNA of P0 worms fed $\mathrm{SAM}-\mathrm{C}^{3} \mathrm{H}_{3}$ (blue bars) and their $\mathrm{F} 1$ progeny (orange bars) as detected by scintillation counting. No radioactive signal was detected in worms (gray bars) or their progeny (yellow bars) fed non-radioactive SAM. Each bar represents 5 or 6 experiments for total lysate, 1 experiment for DNA, 1 experiment for lipids, and 8 or 9 experiments for RNA. Each sample was normalized to the amount of material in that sample so different conditions and experiments could be compared. B, Scheme for feeding paradigm to administer tritiated or deuterated SAM to fed or starved P0 L4 worms. C, Increased radioactive signal is detected in the RNA of both the P0 worms as well as their naïve $\mathrm{F} 1$ progeny when the $\mathrm{P} 0$ generation is starved relative to fed $\mathrm{P} 0$ worms and F1 progeny when fed Methionine- $\mathrm{C}^{3} \mathrm{H}_{3}$. Each column represents the mean $\pm \mathrm{SEM}$ of 4 or 6 independent experiments. ns, not significant, $* \mathrm{p}<0.05$ as assessed by one-way ANOVA and Tukey's multiple comparisons test. D, Naïve F1 progeny whose parents were starved display elevated $\mathrm{m}^{6,2} \mathrm{~A} / \mathrm{A}$ levels on the $18 \mathrm{~S}$ rRNA relative to $\mathrm{F} 1$ progeny whose parents were fed as detected by UHPLC-ms/ms. P0 parents were fed Methionine-CD $\mathrm{CD}_{3}$ and RNA was extracted from F1 eggs. This heat map represents the relative fold change for 4 independent experiments, where each experiment is displayed in one column. 

levels on $18 \mathrm{~S}$ rRNA, respectively, relative to empty vector (EV) control knock-down as assessed by UHPLC-ms/ms. There was no significant effect on m6A levels or other methylation marks on the $18 \mathrm{~S}$ rRNA (Fig. S4A). Each bar represents the mean \pm SEM of 12 independent replicates. ns, not significant, $* \mathrm{p}<0.05, * * * \mathrm{p}<0.001$ as assessed by one-way ANOVA and Holm-Sidak's multiple comparisons test. B, bud-23(tm5768) mutant strain displays a decrease in $\mathrm{m}^{7} \mathrm{G} / \mathrm{G}$ and $\mathrm{m}^{6,2} \mathrm{~A} / \mathrm{A}$ levels while showing increased $\mathrm{m}^{6} \mathrm{~A} / \mathrm{A}$ levels on the $18 \mathrm{~S}$ rRNA as assessed by UHPLC$\mathrm{ms} / \mathrm{ms}$. There was no significant effect on other methylation marks on the $18 \mathrm{~S}$ rRNA (Fig. S4B). Each bar represents the mean \pm SEM of 4 independent experiments. $* \mathrm{p}<0.05, * * * * \mathrm{p}<0.0001$ as assessed by paired t test. C, bud-23 WT but not the catalytically inactive mutant G63E/D8K overexpression lines in bud-23(tm5768) mutant worms rescues $18 \mathrm{~S}$ rRNA m ${ }^{7} \mathrm{G}$ methylation levels, as assessed by UHPLC-MS/MS. D, SCARLET reveals that guanosine 1531 in C. elegans $18 \mathrm{~S}$ rRNA is N7-methylated and this methylation is reduced in bud-23(tm5768) mutant worms. Below is quantification of signal intensities. Control oligos which begin with guanosine were in vitro transcribed using $100 \%$ guanosine or $50 \%$ guanosine and $50 \%$ N7-methylguanosine and were run for reference as to where guanosine and $\mathrm{m}^{7} \mathrm{G}$ should run but are not shown in this blot. 18S rRNA methylation at guanosine 1639 in $293 \mathrm{~T}$ and HCT116 human cell lines is used as a positive control. E, SCARLET reveals that adenosines 1735 and 1736 in C. elegans 18S rRNA are N6-dimethylated and this methylation is reduced in bud-23(tm5768) mutant worms and replaced with N6-monomethylation. Control oligos which begin with adenosine or N6monomethylated adenosine were run for reference as to where adenosine and $\mathrm{m}^{6} \mathrm{~A}$ should run but are not shown in this blot. 18S rRNA methylation at adenosine 1850 in 293T and HCT116 human cell lines is used as a positive control. It should be noted that the calculation shown below just depicts the relative intensity of each residue but is not quantitative due to the effects that $\mathrm{m}^{6,2} \mathrm{~A}$ has on Watson-crick basepairing. F, Coomassie staining of SDS-polyacrylamide gel electrophoresis (SDS-PAGE) gel reveals that GST-tagged DIMT-1 WT and E79A mutant proteins migrate at the same location. G, WT GST-tagged DIMT-1 but not the catalytically inactive mutant E79A is able to methylate $18 \mathrm{~S}$ rRNA purified from bud-23 mutant worms, as assessed by UHPLC-ms/ms of deuterated $\mathrm{m}^{6,2} \mathrm{~A}$. Deuterated S-adenosyl methionine was used as the methyl donor to ensure that methylation was added during methylation assays. Each column represents the mean \pm SEM of three independent experiments. $* * p<0.01$ as assessed by paired $\mathrm{t}$ test. H, WT GST-tagged DIMT-1 but not the catalytically inactive mutant E79A is able to methylate an oligo containing the sequence surrounding adenosines 1735 and 1736 in 18S rRNA. This methylation is absent when the nucleosides representing adenosine 1735 and 1736 are replaced with guanosines despite the presence of additional adenosines in the oligo. (Top) the oligo sequence is displayed with adenosines 1735 and 1736 highlighted in red (bottom) each column represents the mean \pm SEM of three independent experiments. ${ }^{*} \mathrm{p}<0.05$ as assessed by paired test.

Fig. 4 Altered translation of genes involved in development, translation, longevity and stress response in response to bud-23 and dimt-1 knockdown and parental starvation A, Principal component analysis of RNA sequencing of four independent biological replicates reveals that bud-23 and dimt-1 knockdown cause a misregulation of similar sets of genes relative to an empty vector (EV) control. B, Venn diagrams display a high degree of overlap between genes which are dysregulated upon bud-23 knockdown to those which are dysregulated upon 
dimt-1 knockdown. C, Principal component analysis of RNA sequencing of six independent biological replicates reveals that parental starvation cause a misregulation of a large number of genes. D, Venn diagrams display overlap between genes which are dysregulated upon bud-23 or dimt-1 knockdown and those genes which become upregulated in response to parental starvation. E, Revigo plots reveal relative enrichment of coordinately dysregulated gene transcription in response to parental starvation and bud-23 and dimt-1 knockdown. Proximity of bubbles reflects the similarity of terms, color intensity represents $p$ value of enrichment, and size of the bubbles reflects how many genes are in the gene set depicted. F, Heat maps of the 1097 differentially ribosome bound transcripts after bud-23 or dimt-1 knockdown reveals a high degree of overlap between transcripts differentially bound in response to knocking down either rRNA methyltransferase. Each column represents an independent biological replicate. $\mathbf{G}$, GO analysis of transcripts that are differentially bound after knockdown of dimt-1 reveals the importance of $18 \mathrm{~S} \mathrm{~m}{ }^{6,2} \mathrm{~A}$ methylation in regulation of development, reproduction, longevity, and response to heat. H, Heat maps of the 436 differentially ribosome bound transcripts after parental starvation. Each of 6 independent biological replicates cluster together. I, Revigo plots reveal relative enrichment of coordinately dysregulated ribosome binding in response to parental starvation and dimt-1 knockdown.

\section{Fig. 5 dimt-1 and bud-23 are required for intergenerational hormesis}

A, Starvation causes a reduction in reproduction in worms including when bud-23 and dimt-1 are knocked down in the parental P0 generation. Each column represents the mean $\pm \mathrm{SEM}$ of 3 independent experiments performed in three plates with 10 worms per plate. After starvation L1 worms were placed on bacteria expressing double stranded RNA directed against bud-23, dimt-1, or an empty vector (EV). Dots are color coded to display matched independent experiments. B, Starvation causes an increase in survival in response to $37^{\circ} \mathrm{C}$ heat stress for 6 hours. Each column represents the mean \pm SEM of 5 independent experiments performed in three plates with 30 worms per plate. After starvation L1 worms were placed on bacteria expressing double stranded RNA directed against bud-23, dimt-1, or an empty vector (EV). Dots are color coded to display matched independent experiments. C, Knock-down of bud-23 and dimt-1 from the P0 decreases the number of progeny per worm in the fed F1 generation, however, this decrease is not further exacerbated by parental. Worms were maintained on bacteria expressing double stranded RNA directed against bud-23, dimt-1, or an empty vector from the L1 stage of the P0 generation and the entirety of the F1 generation. starvation as parental starvation decreases fertility after empty vector control treatment. Each column represents the mean \pm SEM of 3 independent experiments performed in three plates with 10 worms per plate. Dots are color coded to display matched independent experiments. D, Knock-down of bud-23 and dimt- 1 from the P 0 increases the $37^{\circ} \mathrm{C}$ heat stress resistance in the fed F1 generation, however, this increase is not further enhanced by parental starvation as parental starvation increases heat stress resistance after empty vector control treatment. Each column represents the mean \pm SEM of 4 independent experiments performed in three plates with 30 worms per plate. Dots are color coded to display matched independent experiments. E, Starvation causes a reduction in fertility in both WT and bud23(tm5768) mutant worms in the parental P0 generation. Bud-23(tm5768) mutant worms have reduced fertility relative to WT worms. Each column represents the mean \pm SEM of 3 independent experiments performed in three plates with 10 worms per plate. Dots are color coded to display matched independent experiments. F, Starvation causes an increase in survival in response to $37^{\circ} \mathrm{C}$ heat stress in both WT and bud-23(tm5768) mutant worms in the P0 
615 generation. Bud-23(tm5768) mutant worms display increased basal heat stress relative to WT 616 worms and therefore bud-23(tm5768) mutant worms were maintained at $37^{\circ} \mathrm{C}$ for 9 hours to 617 observe significant fatality in bud-23(tm5768) mutants relative to WT worms which were 618 maintained at $37^{\circ} \mathrm{C}$ for 5.5 hours. Each column represents the mean $\pm \mathrm{SEM}$ of 3 independent 619 experiments performed in three plates with 30 worms per plate. Dots are color coded to display matched independent experiments. G, Deletion of bud-23 eliminates the transmission of reduced fertility to naïve F1 progeny whose parents were starved relative to progeny whose parents were fed. Bud-23(tm5768) mutant worms have reduced fertility relative to WT worms, however, this decrease is not further exacerbated by parental starvation as in the WT worms. Each column represents the mean $\pm \mathrm{SEM}$ of 3 independent experiments performed in three plates with 10 worms per plate. Dots are color coded to display matched independent experiments. H, Deletion of bud-23 eliminates the transmission of increased $37^{\circ} \mathrm{C}$ heat stress survival to naïve progeny whose parents were starved relative to progeny whose parents were fed. Bud-23(tm5768) mutant worms display increased heat stress resistance relative to WT worms, however, this increase is not further enhanced by parental starvation as it is in WT worms. Each column represents the mean \pm SEM of 3 independent experiments performed in three plates with 30 worms per plate. Dots are color coded to display matched independent experiments. ns, not significant, $* \mathrm{p}<0.05$, $* * \mathrm{p}<0.01, * * * \mathrm{p}<0.001, * * * * \mathrm{p}<0.0001$ as assessed by one way ANOVA with Tukey's or Holm-Sidak's multiple comparisons test. 
Liberman $\mathrm{N}$ et al 9/27/21 16

\section{Methods:}

\section{Strains used and RNA interference}

The N2 Bristol strain was used as the wildtype background. Worms were grown on OP50-1 bacteria or $\mathrm{dam}^{-} \mathrm{dcm}^{-}$bacteria (NEB C2925) on standard nematode growth medium (NGM) plates (Brenner, 1974) in all experiments save for RNAi experiments. Bacteria expressing dsRNA of bud-23 and dimt-1 were obtained from the Ahringer and Vidal libraries (a gift from T.K. Blackwell). Bacteria were grown at $37^{\circ} \mathrm{C}$ and seeded on NGM plates containing ampicillin (100 $\mathrm{mg} \mathrm{m}^{-1}$ ) and isopropylthiogalactoside (IPTG; $0.4 \mathrm{mM}$ ). Each vector was sequenced to verify the presence of the appropriate gene of interest. bud-23(tm5768) strain was a gift from Shouhong Guang and was backcrossed 6 times.

\section{Metabolic labeling}

Gravid adult worms were collected in M9 buffer $\left(22 \mathrm{mM} \mathrm{KH}_{2} \mathrm{PO}_{4}, 42 \mathrm{mM} \mathrm{Na}_{2} \mathrm{HPO}_{4}, 86 \mathrm{mM}\right.$ $\mathrm{NaCl}, 1 \mathrm{mM} \mathrm{MgSO} 4)$, washed several times in M9 buffer followed by bleaching $(10 \mathrm{~N} \mathrm{NaOH}$, $\mathrm{NaHOCl}, \mathrm{H} 20$ at a 1:1:11.5 ratio) for egg extraction. Eggs were washed thoroughly several times with either M9 buffer or sterile water and plated on the desired food source. When worms reached L4 stage of development ( 48 hours at $20^{\circ} \mathrm{C}$ ), their food source was replaced with concentrated heat killed bacteria and the desired metabolic label. Metabolic labeling was performed by adding either $\mathrm{SAM}-{ }^{3} \mathrm{H}_{3}$ or Methionine- ${ }^{3} \mathrm{H}_{3}$ (PerkinElmer) at $100-165 \mu \mathrm{Ci}$ or 62.5 mM SAM-D 3 (CDN isotopes) or $250 \mathrm{mM}$ Methionine-D3 (Sigma) to the concentrated bacteria. Unmodified SAM and Methionine were used as negative controls to ensure incorporation occurred during the experiment. It has been shown that SAM is relatively unstable (Morana et al., 2002; Parks and Schlenk, 1958) and therefore it is most likely that any tritium detected in the progeny would have been incorporated into heritably methylated material in the parents and transmitted to the progeny rather than taken up by the progeny themselves or transmitted in the form of SAM- ${ }^{3} \mathrm{H}_{3}$ to be used by the progeny themselves. However, this is still a possibility and that is why subsequent genetic experiments demonstrating the requirement of bud-23 and dimt-1 help to further solidify the findings. Worms were allowed to continue development until day 1 of egg laying. Worms were removed from plates with M9 buffer and eggs were removed from the plate by using a cell scraper and resuspending in M9 buffer. Worms and eggs were washed several times with M9 buffer. Eggs and any remaining worms were bleached twice followed by several washes in water. Worms were washed twice with $70 \%$ Ethanol followed by several washes in water. Worm and Egg samples were flash frozen until processing. For starvation experiments, bleached eggs were plated on NGM plates without food where they hatched and arrested at L1 for 7 days (starved). In parallel a portion of the eggs were plated on NGM with food (fed). Following 7 days the fed population of worms were bleached to extract eggs that were plated on food while the L1 arrested worms were transferred to plates with food 3-4 hours later (initial experiments showed that this allowed both populations to reach the L4 stage when labeling occurs at the same time). Metabolic labeling and collection of the samples occurred as detailed above. Each sample was normalized to the total amount of specific material (DNA, RNA, proteins, lipids) to allow for comparisons between independent measurements. Phenotypic assays following starvation paradigm are detailed below.

\section{Worm lysis and protein quantification}

Worm or egg pellets were resuspended in a homemade lysis buffer $\left(20 \mathrm{mM} \mathrm{NaPO}_{4}, 150 \mathrm{mM}\right.$ $\mathrm{NaCl}, 1 \% \mathrm{NP}-40,0.5 \%$ DOC, $0.5 \%$ SDS, 2 mM EDTA) supplemented with a protease inhibitor 
cocktail (Roche) and $1 \mathrm{mM}$ DTT, followed by 6-8 freeze thaw cycles in liquid nitrogen and an incubation at $95{ }^{\circ} \mathrm{C}$ for 10 minutes. Lysates were cleared by centrifugation at 20,000 $\mathrm{g}$ for 10 min at $4{ }^{\circ} \mathrm{C}$. Protein quantification was performed by Bradford (Bio-Rad) or BCA (ThermoFischer) assay.

\section{$R N A$ extraction}

RNA from worm and egg samples was extracted either with PureLink RNA Mini Kit (Invitrogen) or Direct-zol RNA kit (Zymo). The worm and egg pellets were resuspended in lysis buffer (either homemade supplemented lysis buffer or the kit lysis buffer) or $1 \mathrm{ml}$ of Trizol, followed by 6-8 freeze thaw cycles in liquid nitrogen. RNA was then extracted according to the manufacturer's protocol. As part of the PureLink kit, samples were homogenized with the homogenizer column (Invitrogen). RNA quantification was performed on either a DeNovix DS$11+$ spectrophotometer or a Qubit 3 fluorometer (Invitrogen). To isolate 26S, 18S, and 5.8S/5S rRNAs, total RNA was electrophoresed on agarose gels to separate rRNAs which were excised and purified using either Zymoclean Gel RNA Recovery Kit (Zymo) or ethanol precipitation.

\section{DNA extraction}

Worms and eggs were resuspended in the supplemented homemade lysis buffer, followed by 6-8 freeze thaw cycles in liquid nitrogen. DNA was then extracted using PureLink Genomic DNA Mini Kit (Invitrogen) according to the manufacturer's protocol. DNA quantification was performed on either a DeNovix DS-11+ spectrophotometer or a Qubit 3 fluorometer (Invitrogen).

\section{Lipid extraction}

Worm or egg samples were first lysed according to the protocol detailed above and equal volumes of sample were taken for lipid extraction using a Lipid Extraction kit (BioVision, K216) according to the manufacturer's protocol.

\section{Scintillation counting}

Tritium signal was detected by direct addition of the tested sample (RNA, DNA, lipids, lysate) to Econo-Safe (RPI) followed by scintillation counting on a scintillation counter.

\section{UHPLC-ms/ms}

DNA samples ranging from $500 \mathrm{ng}-2 \mu \mathrm{g}$ were digested to free nucleosides using 5-15 U of DNA Degradase Plus (Zymo Research) in $25 \mu \mathrm{l}$ reactions incubated for $2 \mathrm{hrs}$ at $37^{\circ} \mathrm{C}$. For quantification, pure 2'deoxyadenosine (dA) and N6-methyl-2'-deoxyadenosine (6mdA) nucleosides were used as calibration standards. Quantification was performed as in (Boulias and Greer, 2021), briefly digested samples or pure nucleoside standards were diluted to $100 \mu$ with $\mathrm{ddH} 2 \mathrm{O}$ and filtered through $0.22 \mu \mathrm{m}$ Millex syringe filters and $5 \mu \mathrm{l}$ of the filtered solution was injected for UHPLC-ms/ms analysis, and analyzed using the Agilent 1290 UHPLC system with a C18 reversed-phase column $(2.1 \times 50 \mathrm{~mm}, 1.8 \mathrm{~m})$. Mobile phase A consisted of water with $0.1 \%$ $(\mathrm{v} / \mathrm{v})$ formic acid and mobile phase B consisted of methanol with $0.1 \%(\mathrm{v} / \mathrm{v})$ formic acid. Mass spectrometry detection was performed using an Agilent 6470 triple quadrupole mass spectrometer in positive electrospray ionization mode and data were quantified in dynamic multiple reaction monitoring ( $\mathrm{dMRM}$ ) mode, by monitoring the mass transitions $252.1 \rightarrow 136.0$ for $\mathrm{dA}$ and $266.1 \rightarrow 150.0$ for $6 \mathrm{mdA}$. The ratio of $6 \mathrm{mdA} / \mathrm{A}$ was quantified using calibration 
curves from serial dilutions of pure 6mdA or dA standards. As a negative control in each UHPLC-ms/ms experiment, we included a "mock" digestion reaction, consisting of DNA Degradase Plus and digestion buffer in water, without any added DNA.

To quantify the concentrations of $\mathrm{m}^{6} \mathrm{~A}, \mathrm{~m}^{6,2} \mathrm{~A}, \mathrm{~m}^{7} \mathrm{G}$ and $\mathrm{m}^{5} \mathrm{C}$ in C. elegans RNA samples, we used pure nucleosides of adenosine $(\mathrm{A})$, cytidine $(\mathrm{C})$, guanosine $(\mathrm{G})$, N6-methyladenosine $\left(\mathrm{m}^{6} \mathrm{~A}\right)$, N6-dimethyladenosine $\left(\mathrm{m}^{6,2} \mathrm{~A}\right)$, C5-methylcytidine $\left(\mathrm{m}^{5} \mathrm{C}\right)$, and N7-methylguanosine $\left(\mathrm{m}^{7} \mathrm{G}\right)$ as calibration standards. For digestion to nucleosides, $250 \mathrm{ng}-1 \mu \mathrm{g}$ of RNA samples were digested with Nucleoside Digestion mix (NEB, M069S) for $2 \mathrm{hr}$ at $37^{\circ} \mathrm{C}$. Digested RNA samples or pure nucleoside standards were diluted to $100 \mu \mathrm{l}$ with ddH20 and filtered through $0.22 \mu \mathrm{m}$ Millex Syringe Filters. $5 \mu \mathrm{l}$ of the filtered solution was injected for LC-MS/MS analysis, and analyzed using the Agilent 1290 UHPLC system with a Hypersil Gold C18 reversed-phase column $(2.1 \times 150 \mathrm{~mm}, 3 \mu \mathrm{m})$ as per (Su et al., 2014) with modifications listed below. Mobile phase A consisted of water with $0.1 \%(\mathrm{v} / \mathrm{v})$ formic acid and mobile phase B consisted of acetonitrile with $0.1 \%(\mathrm{v} / \mathrm{v})$ formic acid. Mass spectrometry detection was performed using an Agilent 6470 triple quadrupole mass spectrometer in positive electrospray ionization mode and data were quantified in dynamic multiple reaction monitoring (dMRM) mode, by monitoring the mass transitions $268 \rightarrow 136$ for Adenosine (A), $282 \rightarrow 150$ for N6methyladenosine $\left(\mathrm{m}^{6} \mathrm{~A}\right), 285 \rightarrow 153$ for deuterated N6-methyladenosine $\left(\mathrm{d} 3-\mathrm{m}^{6} \mathrm{~A}\right), 244 \rightarrow 112$ for Cytidine $(\mathrm{C}), 261 \rightarrow 129$ for deuterated C5-methylcytidine $\left(\mathrm{d} 3-\mathrm{m}^{5} \mathrm{C}\right), 284 \rightarrow 152$ for Guanosine (G), $282 \rightarrow 136$ for 2'-O-methyladenosine $(\mathrm{Am}), 285 \rightarrow 136$ for deuterated 2'-Omethyladenosine $(\mathrm{d} 3-\mathrm{Am}), 258 \rightarrow 112$ for 2'-O-methylcytidine $(\mathrm{Cm}), 261 \rightarrow 112$ for deuterated 2'-O-methylcytidine (d3-Cm), $298 \rightarrow 152$ for 2'-O-methylguanosine (Gm), $301 \rightarrow 152$ for deuterated 2'-O-methylguanosine (d3-Gm), $296 \rightarrow 164$ for N6'-N6-dimethyladenosine (m62A), $302 \rightarrow 170$ for deuterated N6'-N6-dimethyladenosine (d3-m62A), $298 \rightarrow 166$ for N7methylguanosine $(\mathrm{m} 7 \mathrm{G}), 301 \rightarrow 169$ for deuterated $\mathrm{N} 7-$ methylguanosine $(\mathrm{d} 3-\mathrm{m} 7 \mathrm{G}), 285 \rightarrow 153$ for deuterated N1-methyladenosine $\left(\mathrm{d} 3-\mathrm{m}^{1} \mathrm{~A}\right)$. The ratio of methylated $\mathrm{A}\left(\% \mathrm{~m}^{6} \mathrm{~A}\right.$ or $\left.\% \mathrm{~m}^{6,2} \mathrm{~A}\right)$ or $\mathrm{G}\left(\% \mathrm{~m}^{7} \mathrm{G}\right)$ in RNA samples was quantified using calibration curves from serial dilutions of the pure ribonucleoside standards.

\section{Recombinant protein}

The coding sequence of dimt- 1 was cloned as an in-frame fusion to the GST tagged vector pGEX-4T1. The catalytic site was mutated through site-directed mutagenesis. The recombinant proteins were expressed in E. coli BL21. Overnight induction of protein expression was carried out with $1 \mathrm{mM}$ IPTG at $18^{\circ} \mathrm{C}$. Bacteria were harvested at $4000 \mathrm{rpm}, 4^{\circ} \mathrm{C}$ and $10 \mathrm{~mL}$ protein purification lysis buffer $(50 \mathrm{mM} \mathrm{pH} 7.5$ Tris- $\mathrm{HCl}, 0.25 \mathrm{M} \mathrm{NaCl}, 0.1 \%$ Triton-X, $1 \mathrm{mM}$ PMSF, 1 $\mathrm{mM}$ DTT, and protease inhibitors). After freezing the pellet at $-80^{\circ} \mathrm{C}$ for 1 hour, the lysate was sonicated with a Bioruptor for 5 minutes on high level with 30 seconds on and 30 seconds off. Proteins were purified with glutathione Sepharose 4B beads. Proteins and beads were washed 3 times with protein purification lysis buffer before incubating the beads with elution buffer (12 $\mathrm{mg} / \mathrm{ml}$ Glutathione in protein purification lysis buffer, $\mathrm{pH} 8.0$ ) for 30 minutes. Eluates were dialyzed overnight at $4^{\circ} \mathrm{C}$ with dialysis buffer $(50 \mathrm{mM} \mathrm{pH} 8.0$ Tris- $\mathrm{HCl}, 1 \mathrm{mM}$ EDTA, $1 \mathrm{mM}$ DTT, and 20\% glycerol). Bradford assays and SDS-page gel electrophoresis followed by coomassie staining was performed to determine integrity and quantity of purified proteins. 
In vitro methylation reactions assaying methyltransferase activity of dam or HpaII (NEB) on DNA were performed in the buffer supplied with the commercial recombinant enzyme (New England Biolabs (NEB) dam Methyltransferase Reaction Buffer or CutSmart Buffer) per the NEB protocol. Methyltransferase activity was assessed on $0.5-2 \mu \mathrm{g}$ of pL4440 plasmid DNA extracted from dam $\mathrm{dcm}^{-}$bacteria. In vitro reactions were performed with $80 \mu \mathrm{M}$ or $160 \mu \mathrm{M}$ SAM-D 3 or a mixture of SAM and SAM-D 3 as indicated. Reactions were purified using a PCR purification kit (Invitrogen) followed by digestion with DNA degradase plus (Zymo) for UHPLC-MS/MS analysis. For radioactive in vitro assays, $0.4 \mu \mathrm{M}$ or $3.2 \mu \mathrm{M}$ of $\mathrm{SAM}_{-}{ }^{3} \mathrm{H}_{3}$ were used and the reaction was cleaned with either a PCR purification kit or Bio-Spin P30 columns (Bio-rad). The reactions were incubated for $2 \mathrm{hrs}$ at $37^{\circ} \mathrm{C}$, followed by enzyme deactivation for 20 minutes at $65^{\circ} \mathrm{C}$. In vitro reactions with GST-DIMT-1 were performed as in (Shen et al., 2020 ), briefly $30 \mu \mathrm{l}$ reactions containing $2 \mu \mathrm{g}$ of $18 \mathrm{~S}$ rRNA or oligos were incubated with $12 \mu \mathrm{gs}$ of DIMT-1 WT or E79A mutant, $1 \mathrm{mM} \mathrm{d}$-SAM, $50 \mathrm{mM}$ Tris $\mathrm{pH} 7.5,5 \mathrm{mM} \mathrm{MgCl}_{2}$, and $1 \mathrm{mM}$ DTT at $16^{\circ} \mathrm{C}$ overnight. Then reactions were incubated for 20 minutes at $65^{\circ} \mathrm{C}$, followed by clean up and buffer exchange with Bio-Spin P30 columns (Bio-rad). RNA was digested to nucleosides with 20 units of S1 Nuclease (ThermoScientific) at $37^{\circ} \mathrm{C}$ for 2 hours followed by treatment with Fast Alkaline Phosphatase (ThermoScientific) for 1 hour at $37^{\circ} \mathrm{C}$. Samples were diluted $2 \mathrm{X}$ with milliQ water and $5 \mu \mathrm{l}$ were used for UHPLC-MS/MS analysis. Synthesized 18S rRNA oligos of the following sequences: A1735, 1736: GCUGUAGGUGAACCUGCAGCUGG and A1735,1736->G: GCUGUAGGUGGGCCUGCAGCUGG were obtained from IDT.

Site-specific cleavage and radioactive-labeling followed by ligation assisted extraction and thinlayer chromatography (SCARLET)

SCARLET assays were performed as in (Liu et al., 2013). Briefly, in the first step 18S rRNA was subjected to RNAse H site-specific cleavage directed by 2'-O-methyl RNA-DNA chimeras with the following sequences; $C$. elegans $18 \mathrm{~S}$ rRNA G1531 chimeric oligo: 5'mGmGmCmAmUmUmCCTCGmUmUmUmAmAmGmG-3', C. elegans 18S rRNA A1735 chimeric oligo: 5' - mGmCmAmGmGmUmUCACCmUmAmCmAmGmCmU-3', C. elegans 18S rRNA A1736 chimeric oligo: 5'- mUmGmCmAmGmGmUTCACmCmUmAmCmAmGmC-3', H. sapiens 18S rRNA G1639 chimeric oligo: 5'-

mGmGmAmAmUmUmCCTCGmUmUmCmAmUmGmG-3', H. sapiens 18S rRNA A1850 chimeric oligo: 5' - mGmCmAmGmGmUmUCACCmUmAmCmGmGmAmA-3'. 200 ng of gel purified 18S rRNA was mixed with 5 pmoles chimeric oligo in $30 \mathrm{mM}$ Tris-HCL, $\mathrm{pH}=7.5$ in a total volume of $5 \mu \mathrm{l}$. The resulting mixture was heated for $3 \mathrm{~min}$ at $95^{\circ} \mathrm{C}$ followed by cooling to RT for 3 min. RNAse H (5 Units, NEB), rSAP (1 Unit, NEB) and RNasin (20 units, Promega) were added in a total volume of $10 \mu \mathrm{l}$ in $1 \mathrm{X}$ T4 PNK buffer (NEB) and the mixture was incubated for $1 \mathrm{hr}$ at $44^{\circ} \mathrm{C}$, followed by heat inactivation for $5 \mathrm{~min}$ at $75^{\circ} \mathrm{C}$. Radioactive endlabeling was performed with the addition of T4 PNK (20 Units, NEB) and $2 \mu 1\left[\gamma-{ }^{32} \mathrm{P}\right] \mathrm{ATP}$ $(6000 \mathrm{Ci} / \mathrm{mmol})$ at $37^{\circ} \mathrm{C}$ for $1 \mathrm{hr}$ in a total volume of $15 \mu \mathrm{l}$ in $1 \mathrm{X} \mathrm{T} 4 \mathrm{PNK}$ buffer, followed by heat inactivation for $5 \mathrm{~min}$ at $75^{\circ} \mathrm{C}$. The free $\left[\gamma^{32} \mathrm{P}\right] \mathrm{ATP}$ was removed by the use of Bio-Spin 6 column (Biorad) according to the manufacturer's instructions. The radioactive labeled $18 \mathrm{~S}$ fragments were subjected to splint ligation by the addition of 5 pmoles splint oligo and 5 pmoles of 116-mer ssDNA oligo of the following sequences; C. elegans 18S rRNA G1531 splint oligo: 5 -

AGCTGATGACTCACACTTACTAGGCATTCCTATTAACTCACAGGACCGGCGATGGCT G-3', C. elegans 18S rRNA A1735 splint oligo: 5'- 
CGATGATCCAGCTGCAGGTTCTATTAACTCACAGGACCGGCGATGGCTG -3', $C$. elegans 18S rRNA A1736 splint oligo: 5'CGATGATCCAGCTGCAGGTCTATTAACTCACAGGACCGGCGATGGCTG-3', H. sapiens 18S rRNA G1639 splint oligo: 5'AGCTTATGACCCGCACTTACTGGGAATTCCTATTAACTCACAGGACCGGCGATGGCT G-3', H. sapiens 18S rRNA A1850 splint oligo: 5'TAATGATCCTTCCGCAGGTTCTATTAACTCACAGGACCGGCGATGGCTG-3', 116-mer ssDNA oligo: 5'TTGACTTAAAGTCTAACCTATAGGATACTTACAGCCATCGCCGGTCCTGTGAGTTAA TAG-3'. The resulting mixture was heated for $3 \mathrm{~min}$ at $75^{\circ} \mathrm{C}$ followed by cooling to RT for 3 min. Ligation was performed in a total volume of $20 \mu \mathrm{l}$ by the addition of $1 \mu \mathrm{l} 4$ DNA Ligase (400 Units, NEB) in 1X T4 DNA Ligase buffer (NEB) and the mixture was incubated for $3 \mathrm{hr}$ at $37^{\circ} \mathrm{C}$. RNA was degraded by the addition of $1 \mu \mathrm{RNAseA} / \mathrm{T} 1 \mathrm{mix}$ (Thermo) for $1 \mathrm{hr}$ at $37^{\circ} \mathrm{C}$ and the ligation reaction was stopped by the addition of $2 \mu 1500 \mathrm{mM}$ EDTA and $20 \mu \mathrm{l}$ Novex $2 \mathrm{X}$ TBE-Urea Sample buffer (Thermo). The radioactive ligation mixtures were subjected to TBEurea gel electrophoresis followed by staining with SYBR gold. The band that corresponded to the radiolabeled splint ligated $117 / 118 \mathrm{bp}$ fragment was excised and was eluted for $3 \mathrm{hr}$ at $37^{\circ} \mathrm{C}$ in $300 \mu \mathrm{l}$ gel extraction buffer (300 mM NaOAc pH5.5, $1 \mathrm{mM}$ EDTA, 0.25\% $/ \mathrm{v}$ SDS), followed by ethanol precipitation. The purified fragment was resuspended in DEPC-treated water and was digested with Nuclease P1 (2 Units, Wako USA) in $10 \mathrm{mM}$ ammonium acetate $\mathrm{pH}=5.2,2 \mathrm{mM}$ $\mathrm{ZnCl}_{2}$ for $2 \mathrm{hr}$ at $60^{\circ} \mathrm{C}$ in a total volume of $20 \mu \mathrm{l} .2 .5 \mu \mathrm{l}$ of the digested nucleotide mixture was analyzed by TLC on a glass-backed PEI-cellulose plate (Merck Millipore) in a buffer containing isopropanol/HCl/water (70:15:15). Signal acquisition and quantification of the radiolabeled adenosine and N6-methyladenosine, N6-dimethylated adenosine, Guanosine and N7methylguanosine was carried out using a BAS storage phosphor screen (GE Healthcare Life Sciences) at $200 \mu \mathrm{m}$ resolution using the ImageQuantTL software (GE Healthcare Life Sciences).

\section{Lifespan assays}

Worm lifespan assays were performed at $20^{\circ} \mathrm{C}$, without 5-fluoro-2'-deoxyuridine (FUDR), as described previously (Greer et al., 2007) unless noted otherwise. For each lifespan assay, $\sim 90$ worms per condition were used in three plates to begin the experiment ( 30 worms per plate). Worms that underwent matricide, exhibited a ruptured vulva, or crawled off the plates were censored. Statistical analysis of lifespan were performed on Kaplan-Meier survival curves in Prism 8.4.3 by log rank (Mantel-Cox) tests. The values from the Kaplan-Meier curves are included in Supplementary Table 1.

\section{Heat stress assays}

Synchronized L4 worms were placed at $37^{\circ} \mathrm{C}$ for the time indicated and then grown at $20^{\circ} \mathrm{C}$ for the remainder of the assay. Each experiment included at least 30 worms per plate with three plates per condition. Survival was assessed every $24 \mathrm{hrs}$ after initial heat stress.

$25^{\circ} \mathrm{C}$ heat stress assays were performed as in (Klosin et al., 2017; Schott et al., 2014). Briefly, embryos were extracted from gravid adults as described above and plated on NGM plates with food incubated at $20^{\circ} \mathrm{C}$ or $25^{\circ} \mathrm{C}$. Plates with eggs were incubated either at $20^{\circ} \mathrm{C}$ or $25^{\circ} \mathrm{C}$. Worm 
populations that reached the $\mathrm{L} 4$ stage (worms at $25^{\circ} \mathrm{C}$ reached $\mathrm{L} 4$ sooner than worms at $20^{\circ} \mathrm{C}$ ) were metabolically labeled and either returned to $20^{\circ} \mathrm{C}$ or shifted from $25^{\circ} \mathrm{C}$ to $20^{\circ} \mathrm{C}$.

\section{Fertility assays}

From day 3 to day 8 post-hatching, 10 worms were placed on NGM plates with dam ${ }^{-} \mathrm{dcm}^{-}$ bacteria in triplicate (30 worms total per condition). Worms were grown at $20^{\circ} \mathrm{C}$. After $24 \mathrm{hrs,}$ the adult worms were removed from each plate and placed on new plate. The numbers of eggs and hatched worms on the plate were counted. Statistical analyses of fertility were performed using t-tests using mean and standard error values.

\section{Ribosome profiling}

Ribosome profiling was performed according to published protocol (Aeschimann et al., 2015) with modifications according to published protocols (Gerashchenko and Gladyshev, 2017). Flash frozen worm pellets were lysed and homogenized in lysis buffer $(20 \mathrm{mM}$ Tris-HCl pH 7.5, $50 \mathrm{mM}$ $\mathrm{KCl}, 50 \mathrm{mM} \mathrm{NaCl}, 5 \mathrm{mM} \mathrm{MgCl}$, $100 \mu \mathrm{g} / \mathrm{ml}$ Cycloheximide, $1 \mathrm{mM}$ DTT, EDTA-free protease inhibitors cocktails (Roche), 1\% Triton X100) using pellet pestles for $1.7 \mathrm{ml}$ tubes. $10 \%$ of each lysate was immediately taken to isolate total RNA by adding $300 \mu \mathrm{l}$ of Trizol-LS (Invitrogen) and proceeding with Direct-Zol miniprep kit (Zymo). $\mathrm{CaCl}_{2}$ was added to a final concentration of 5 $\mathrm{mM}$ to the rest of the lysate. Lysates were treated with $600 \mathrm{U}$ of RNase S7 (Roche) for 1 hour at room temperature. RNA digestion was quenched by supplementing $10 \mu \mathrm{l}$ of $0.5 \mathrm{M}$ EGTA. Treated lysates were run on sucrose gradients (10-50\%) and the monosome peak was collected and concentrated on $100 \mathrm{kDa}$ Amicon filter columns (Millipore). RNA from the monosome fraction was extracted using TRIzol LS and a Direct-zol kit (Zymo). The RNA was loaded on a Novex $15 \%$ TBE-Urea gel (Life Technologies) and a range of fragments between 25 and 32 bps were excised and eluted from the gel. The library was prepared using the TruSeq Small RNA kit (Illumina) according to published protocol (Aeschimann et al., 2015). The PCR product was then loaded on a Novex 6\% TBE-Urea gel (Life Technologies) and a band around 160-170 bp was excised from the gel. The DNA was eluted from the gel and sent for quality assurance and sequencing at the Biopolymers facility at Harvard University. RNA for mRNA sequencing was extracted using Direct-zol and sent for polyA selection, library preparation and sequencing at Novogene Inc.

\section{Transcriptome and ribosome profiling sequencing and analysis.}

Transcriptomes and ribosome profiling libraries were sequenced on the Illumina NovaSeq 6000 and NextSeq 500 platforms. mRNA libraries were sequenced in a paired-end mode with each read being 150 nucleotides long. Ribosome profiling libraries were sequenced in a single-end mode with 51 nucleotides read length before adapter trimming. Adapters were removed with Cutadapt software (Martin, 2011), short reads alignment and counting performed with STAR aligner (Dobin et al., 2013). Differential gene expression was evaluated with the DESeq 2 package in the $\mathrm{R}$ programming environment (Love et al., 2014). Gene set enrichment analysis was done with GSEA stand-alone software (Broad Institute, (Subramanian et al., 2005)) using a collection of C. elegans gene lists derived from the gene2go annotation data at the NCBI. They are analogous to the GObased series of human-only collections available from MSigDB: a source gene list collection used in the original implementation of GSEA software by Broad Institute. 
Fluorescent GFP bacteria consumption

916 Starvation assays were set up as described above. Worms that reached the L4 stage were

917 transferred to plates spotted with OP50-GFP bacteria to feed for $2 \mathrm{hrs.} 30$ worms were then

918 moved to plates without food for 5 minutes. 10 worms were transferred to $2 \%$ agar slides with a

919 drop of $50 \mathrm{mM} \mathrm{NaN3}$ as a paralytic. GFP detection was performed on a Zeiss Discovery V8

920

921

922 fluorescent microscope. GFP fluorescence was quantified using ImageJ.

923

Transgenic strain creation

924

Expression vectors for creating transgenic strains were based on pSD1 plasmid vector (a gift

925 from W. Mair and S. Dutta) that contains the ubiquitous eft-3 promoter and unc-54 3'

926 untranslated region. Bud-23 and the bud-23 G63E/D82K catalytic mutant were amplified from

927 the pGEX-4T1 constructs, followed by restriction-free cloning into the pSD1. Germline transformation experiments were performed as described (Mello et al., 1991). For the bud-23 rescue experiments, injection mixes contained $\mathrm{pSD} 1::$ bud-23 or pSD1::bud-23 G63E/D82K plasmids at $50 \mathrm{ng} / \mu \mathrm{l}, \mathrm{pTG} 96\left(20 \mathrm{ng} / \mu \mathrm{l} ; P_{S u r-5}: \because g f p\right)$ as a cotransformation marker, and 1-kb DNA ladder (80 ng/ $\mu$; Invitrogen) as carrier DNA. 
972

973

974

975

976

\section{Supplemental information:} Fig. S1 Starvation induces transgenerational reduced fertility and increased heat stress resistance that revert in the $\mathrm{F} 3$ generation

A, Naïve F2 progeny whose grandparents were starved have reduced fertility relative to progeny whose grandparents were fed. Each column represents the mean \pm SEM of 3 independent experiments performed in three plates with 10 worms per plate. Dots are color coded to display matched independent experiments. ${ }^{*} \mathrm{p}<0.05$ as assessed by unpaired $t$ test. B, Naïve F2 progeny whose grandparents were starved display an increase in survival in response to $37^{\circ} \mathrm{C}$ heat stress for 6 hours relative to progeny whose grandparents were fed. Each column represents the mean \pm SEM of 3 independent experiments performed in three plates with 30 worms per plate. Dots are color coded to display matched independent experiments. ${ }^{*} \mathrm{p}<0.05$ as assessed by two-way ANOVA. C, Naïve F3 progeny whose great grandparents were starved have similar fertility relative to progeny whose great grandparents were fed. Each column represents the mean \pm SEM of 3 independent experiments performed in three plates with 10 worms per plate. Dots are color coded to display matched independent experiments. ns, not significant as assessed by unpaired $\mathrm{t}$ test. D, Naïve F3 progeny whose great grandparents were starved do not display an increase in survival in response to $37^{\circ} \mathrm{C}$ heat stress for 6 hours relative to progeny whose great grandparents were fed. Each column represents the mean \pm SEM of 3 independent experiments performed in three plates with 30 worms per plate. Dots are color coded to display matched independent experiments. ns, not significant as assessed by two-way ANOVA.

Fig. S2 Deuterated and tritiated SAM can be utilized by enzymes with equal activity and starved descendants eat similar amounts of OP50 to fed descendants

A, The C5-cytosine methyltransferase HpaII can utilize tritiated SAM to methylate DNA as detected by scintillation counting. B, The DNA adenine methylase dam can utilize deuterated SAM to methylate DNA as assessed by UHPLC-ms/ms. As increasing concentrations of deuterated SAM were incubated with dam and DNA there was an increased relative incorporation of deuterated N6-methyladenosine relative to hydrogen methyl groups. SAM was used more efficiently than deuterated SAM. The numbers in the $\mathrm{x}$ axis represent the relative amount of [SAM] to [SAM-D $]$. $\mathbf{C}$, Increased radioactive signal is detected in the RNA of both the P0 worms as well as their naïve F1 progeny when the P0 generation is starved relative to fed $\mathrm{P} 0$ worms and F1 progeny when fed SAM-C $\mathrm{C}^{3} \mathrm{H}_{3}$. Each column represents the mean $\pm \mathrm{SEM}$ of 4 or 5 independent experiments. D, Starved worms do not consume more food than fed worms after recovering on food for 2 days as assessed by GFP fluorescence in the intestine of worms fed OP50 expressing GFP.

Fig. S3 Heat stress causes no consistent heritable change in methylation

A, After a $25^{\circ} \mathrm{C}$ heat stress there was no increase in radioactive methyl groups incorporated into P0 parental worms or their F1 naïve descendants as assessed by scintillation counting of total lysate using tritiated SAM as the methyl donor. B, After a $25^{\circ} \mathrm{C}$ heat stress there was no increase in radioactive methyl groups incorporated into RNA of P0 parental worms or their F1 naïve descendants as assessed by scintillation counting using tritiated SAM as the methyl donor.

Fig. S4 There is no change in 2'O methylation modifications on the 18S rRNA after deletion of bud-23 or knock-down of bud-23 or dimt-1 
A, Knock-down of dimt-1 and bud-23 caused no change in $\mathrm{A}_{\mathrm{m}} / \mathrm{A}, \mathrm{G}_{\mathrm{m}} / \mathrm{G}$, or $\mathrm{C}_{\mathrm{m}} / \mathrm{C}$ levels on $18 \mathrm{~S}$ rRNA relative to empty vector (EV) control knock-down as assessed by UHPLC-ms/ms. Each bar represents the mean \pm SEM of 12 independent replicates. ns, not significant as assessed by one-way ANOVA with Dunnett's multiple comparison test. B, bud-23(tm5768) mutant strain displays no change in $\mathrm{A}_{\mathrm{m}} / \mathrm{A}, \mathrm{G}_{\mathrm{m}} / \mathrm{G}$, or $\mathrm{C}_{\mathrm{m}} / \mathrm{C}$ levels on $18 \mathrm{~S}$ rRNA relative to WT control worms as assessed by UHPLC-ms/ms. Each bar represents the mean \pm SEM of 4 independent experiments. ns, not significant as assessed by paired t test.

Fig. S5 F1 eggs from A, fed and B, starved parents have similar polysome profiles Polysome profiles of descendants from (A) fed parents were indistinguishable from polysome profiles of descendants from (B) starved parents. This graph is a representative experiment where $\mathrm{UV}$ absorbance at $\mathrm{OD}_{254}$ (optical density at $254 \mathrm{~nm}$ ) is monitored continuously.

Fig. S6 Knock down of bud-23 or dimt-1 causes a similar dysregulation of gene expression as parental starvation $\mathbf{A}$, Scatter plots of RNA sequencing show pair wise comparisons of F1 fed (fe) and starved (st) worms and WT worms after knockdown of bud-23 (bu), dimt-1 (di), or an empty vector $(\mathrm{EV})$ control. $\mathbf{B}, \mathrm{GO}$ analysis of genes which are coordinately differentially transcribed after bud-23 and dimt-1 knockdown reveals the importance of $18 \mathrm{~S}$ methylation in regulation of development, reproduction, longevity, and translation. $\mathbf{C}$, GO analysis of genes which are differentially upregulated after parental starvation reveals an effect on translation, the response to heat and the endoplasmic reticulum unfolded protein response. D, Parental starvation does not affect dimt-1 (left) or bud-23 (right) expression levels. ns, not significant as assessed by t test. E, WormCat gene ontology analysis (Holdorf et al., 2020) reveal relative enrichment of coordinately dysregulated gene transcription in response to parental starvation and bud-23 and dimt-1 knockdown.

Fig. 57 Knock down of bud-23 or dimt-1 causes a similar change in translation efficiency as parental starvation $\mathbf{A}$, Scatter plots of translation efficiency show pair wise comparisons of F1 fed and starved worms and WT worms after knockdown of bud-23, dimt-1, or an empty vector (EV) control. B, Principal component analysis of translation efficiency after knockdown of bud23 and dimt- 1 reveals that bud-23 and dimt- 1 knockdown cause a similar change in binding of the ribosome to transcripts relative to an empty vector $(\mathrm{EV})$ control. $\mathbf{C}$, GO analysis of transcripts that are differentially bound after bud-23 knockdown reveals an effect on pathways involved in regulation of development, reproduction, and longevity. D, GO analysis of transcripts that are coordinately differentially bound after bud-23 and dimt-1 knockdown reveals an effect on pathways involved in regulation of development, growth, regulation of gene expression, and longevity. E, GO analysis of transcripts that are differentially bound after parental starvation reveals an effect on pathways involved in regulation of development, reproduction, longevity, and translation. F, Venn diagram display overlap between dysregulated translation efficiency upon dimt- 1 knockdown and parental starvation. $\mathrm{p}<1 \mathrm{E}-9$ by hypergeometric probability. G, GO analysis of transcripts that are coordinately differentially bound after dimt- 1 knockdown and starvation reveals an effect on pathways involved in regulation of development, reproduction, cellular response to stress, and longevity.

Fig. S8 bud-23 is necessary for parental longevity extension in response to starvation and starved worms fed HT115 bacteria do not display increased lifespan 
1023

1024

1025

1026

1027

1028

1029

1030

1031

1032

1033

1034

1035

1036

1037

1038

1039

1040

1041

1042

1043

1044

1045

1046

1047

1048

1049

1050

1051

1052

1053

\begin{abstract}
A, Starvation causes no significant change in lifespan when both fed and starved worms are placed on HT115 empty vector expressing bacteria. Each condition represents three plates of $\sim 30$ worms per plate. This is a representative experiment which has been performed 2 times. B, Starvation causes a subtle increase in longevity of WT worms but not bud-23(tm5768) mutant worms. Each condition represents three plates of $\sim 30$ worms per plate. This is a representative experiment which has been performed 3 times. Statistics of independent experiments are presented in Supplementary Table 1 . Ns, not significant, ${ }^{*} p<0.05$ as assessed by Log-rank (Mantel-Cox) test. Statistics of independent experiments are presented in Supplementary Table 1 .
\end{abstract}

\title{
Supplementary Table 1. Parental starvation causes an increase in lifespan in the P0 and F1 generation in WT worms but not in bud-23(tm5768) mutant worms. The figure panels in
} which specific experiments are shown or used are indicated in the right column. The mean lifespan and SD values were calculated by Prism from triplicate samples of 30 worms each ( 90 worms total). \# worms: number of observed dead worms at the end of the experiment/number of alive worms at the beginning of the experiment. The difference between both numbers corresponds to the number of censored worms (worms that underwent "matricide", exhibited ruptured vulva, or crawled off the plates). $\mathrm{P}$ values are calculated by log rank (Mantel-Cox) statistical test.

\section{Supplementary Table 2}

The tables shows normalized and log-transformed RNAseq expression values from eggs knockdown of bud-23 or dimt-1 relative to an empty vector (EV) control or in F1 children in response to parental starvation relative to fed parents.

\section{Supplementary Table 3}

The tables shows normalized and log-transformed translation efficiency values from eggs knockdown of bud-23 or dimt-1 relative to an empty vector (EV) control or in F1 children in response to parental starvation relative to fed parents. 
1054

1055

1056

1057

1058

1059

1060

1061

1062

1063

1064

1065

1066

1067

1068

1069

1070

1071

1072

1073

1074

1075

1076

1077

1078

1079

1080

1081

1082

1083

1084

1085

1086

1087

1088

1089

1090

1091

1092

1093

1094

1095

1096

1097

\section{References:}

Aeschimann, F., Xiong, J., Arnold, A., Dieterich, C., and Grosshans, H. (2015). Transcriptomewide measurement of ribosomal occupancy by ribosome profiling. Methods 85, 75-89.

Basu, A., Das, P., Chaudhuri, S., Bevilacqua, E., Andrews, J., Barik, S., Hatzoglou, M., Komar, A.A., and Mazumder, B. (2011). Requirement of rRNA methylation for $80 \mathrm{~S}$ ribosome assembly on a cohort of cellular internal ribosome entry sites. Mol Cell Biol 31, 4482-4499.

Blobel, G., and Potter, V.R. (1967). Studies on free and membrane-bound ribosomes in rat liver. I. Distribution as related to total cellular RNA. J Mol Biol 26, 279-292.

Boskovic, A., and Rando, O.J. (2018). Transgenerational Epigenetic Inheritance. Annu Rev Genet 52, 21-41.

Boulias, K., and Greer, E.L. (2021). Detection of DNA Methylation in Genomic DNA by UHPLCMS/MS. Methods Mol Biol 2198, 79-90.

Boulias, K., Toczydlowska-Socha, D., Hawley, B.R., Liberman, N., Takashima, K., Zaccara, S., Guez, T., Vasseur, J.J., Debart, F., Aravind, L., et al. (2019). Identification of the m(6)Am Methyltransferase PCIF1 Reveals the Location and Functions of m(6)Am in the Transcriptome. Molecular cell 75, 631-643 e638.

Brenner, S. (1974). The genetics of Caenorhabditis elegans. Genetics 77, 71-94.

Cenik, E.S., Meng, X., Tang, N.H., Hall, R.N., Arribere, J.A., Cenik, C., Jin, Y., and Fire, A. (2019). Maternal Ribosomes Are Sufficient for Tissue Diversification during Embryonic Development in C. elegans. Dev Cell 48, 811-826 e816.

Champe, P.C., and Harvey, R.A. (1994). Lippincott's Illustrated Reviews: Biochemistry 2nd edition. Lippincott Williams \& Wilkins.

Cheng, Q., Trangucci, R., Nelson, K.N., Fu, W., Collender, P.A., Head, J.R., Hoover, C.M., Skaff, N.K., Li, T., Li, X., et al. (2020). Prenatal and early-life exposure to the Great Chinese Famine increased the risk of tuberculosis in adulthood across two generations. Proceedings of the National Academy of Sciences of the United States of America 117, 27549-27555.

Daxinger, L., and Whitelaw, E. (2012). Understanding transgenerational epigenetic inheritance via the gametes in mammals. Nature reviews. Genetics 13, 153-162.

Demirci, H., Murphy, F.t., Belardinelli, R., Kelley, A.C., Ramakrishnan, V., Gregory, S.T., Dahlberg, A.E., and Jogl, G. (2010). Modification of $16 \mathrm{~S}$ ribosomal RNA by the KsgA methyltransferase restructures the $30 \mathrm{~S}$ subunit to optimize ribosome function. RNA 16, 2319-2324.

Demoinet, E., Li, S., and Roy, R. (2017). AMPK blocks starvation-inducible transgenerational defects in Caenorhabditis elegans. Proceedings of the National Academy of Sciences of the United States of America 114, E2689-E2698.

Dobin, A., Davis, C.A., Schlesinger, F., Drenkow, J., Zaleski, C., Jha, S., Batut, P., Chaisson, M., and Gingeras, T.R. (2013). STAR: ultrafast universal RNA-seq aligner. Bioinformatics 29, 15-21.

Gaydos, L.J., Wang, W., and Strome, S. (2014). Gene repression. H3K27me and PRC2 transmit a memory of repression across generations and during development. Science (New York, N.Y 345, 1515-1518.

Gerashchenko, M.V., and Gladyshev, V.N. (2017). Ribonuclease selection for ribosome profiling. Nucleic Acids Res 45, e6.

Greer, E.L., Beese-Sims, S.E., Brookes, E., Spadafora, R., Zhu, Y., Rothbart, S.B., AristizabalCorrales, D., Chen, S., Badeaux, A.I., Jin, Q., et al. (2014). A histone methylation network regulates transgenerational epigenetic memory in C. elegans. Cell Reports 7, 113-126. 
1098

1099

1100

1101

1102

1103

1104

1105

1106

1107

1108

1109

1110

1111

1112

1113

1114

1115

1116

1117

1118

1119

1120

1121

1122

1123

1124

1125

1126

1127

1128

1129

1130

1131

1132

1133

1134

1135

1136

1137

1138

1139

1140

Greer, E.L., Dowlatshahi, D., Banko, M.R., Villen, J., Hoang, K., Blanchard, D., Gygi, S.P., and Brunet, A. (2007). An AMPK-FOXO pathway mediates longevity induced by a novel method of dietary restriction in C. elegans. Curr Biol 17, 1646-1656.

Greer, E.L., Maures, T.J., Hauswirth, A.G., Green, E.M., Leeman, D.S., Maro, G.S., Han, S., Banko, M.R., Gozani, O., and Brunet, A. (2010). Members of the H3K4 trimethylation complex regulate lifespan in a germline-dependent manner in C. elegans. Nature 466, 383-387.

Haag, S., Kretschmer, J., and Bohnsack, M.T. (2015). WBSCR22/Merm1 is required for late nuclear pre-ribosomal RNA processing and mediates N7-methylation of G1639 in human $18 \mathrm{~S}$ rRNA. RNA 21, 180-187.

Holdorf, A.D., Higgins, D.P., Hart, A.C., Boag, P.R., Pazour, G.J., Walhout, A.J.M., and Walker, A.K. (2020). WormCat: An Online Tool for Annotation and Visualization of Caenorhabditis elegans Genome-Scale Data. Genetics 214, 279-294.

Houri-Zeevi, L., Korem Kohanim, Y., Antonova, O., and Rechavi, O. (2020). Three Rules Explain Transgenerational Small RNA Inheritance in C. elegans. Cell 182, 1186-1197 e1112.

Houri-Zeevi, L., Teichman, G., Gigold, H., and Rechavi, O. (2019). Stress Resets

Transgenerational Small RNA Inheritance. bioRxiv, https://doi.org/10.1101/669051.

Ingolia, N.T., Ghaemmaghami, S., Newman, J.R., and Weissman, J.S. (2009). Genome-wide analysis in vivo of translation with nucleotide resolution using ribosome profiling. Science (New York, N.Y 324, 218-223.

Islam, K., Zheng, W., Yu, H., Deng, H., and Luo, M. (2011). Expanding cofactor repertoire of protein lysine methyltransferase for substrate labeling. ACS Chem Biol 6, 679-684.

Ito, H., Gaubert, H., Bucher, E., Mirouze, M., Vaillant, I., and Paszkowski, J. (2011). An siRNA pathway prevents transgenerational retrotransposition in plants subjected to stress. Nature 472, 115-119.

Jimenez-Chillaron, J.C., Isganaitis, E., Charalambous, M., Gesta, S., Pentinat-Pelegrin, T., Faucette, R.R., Otis, J.P., Chow, A., Diaz, R., Ferguson-Smith, A., et al. (2009). Intergenerational transmission of glucose intolerance and obesity by in utero undernutrition in mice. Diabetes 58 , 460-468.

Jobson, M.A., Jordan, J.M., Sandrof, M.A., Hibshman, J.D., Lennox, A.L., and Baugh, L.R. (2015).

Transgenerational Effects of Early Life Starvation on Growth, Reproduction, and Stress

Resistance in Caenorhabditis elegans. Genetics 201, 201-212.

Kaletsky, R., Moore, R.S., Vrla, G.D., Parsons, L.R., Gitai, Z., and Murphy, C.T. (2020). C. elegans interprets bacterial non-coding RNAs to learn pathogenic avoidance. Nature 586, 445-451.

Klosin, A., Casas, E., Hidalgo-Carcedo, C., Vavouri, T., and Lehner, B. (2017). Transgenerational transmission of environmental information in C. elegans. Science (New York, N.Y 356, 320-323. Lafontaine, D., Delcour, J., Glasser, A.L., Desgres, J., and Vandenhaute, J. (1994). The DIM1 gene responsible for the conserved m6(2)Am6(2)A dimethylation in the 3'-terminal loop of $18 \mathrm{~S}$ rRNA is essential in yeast. J Mol Biol 241, 492-497.

Lang-Mladek, C., Popova, O., Kiok, K., Berlinger, M., Rakic, B., Aufsatz, W., Jonak, C., Hauser, M.T., and Luschnig, C. (2010). Transgenerational inheritance and resetting of stress-induced loss of epigenetic gene silencing in Arabidopsis. Mol Plant 3, 594-602.

Letoquart, J., Huvelle, E., Wacheul, L., Bourgeois, G., Zorbas, C., Graille, M., Heurgue-Hamard, V., and Lafontaine, D.L. (2014). Structural and functional studies of Bud23-Trm112 reveal 18S 
1141 rRNA N7-G1575 methylation occurs on late 40S precursor ribosomes. Proceedings of the 1142 National Academy of Sciences of the United States of America 111, E5518-5526. Li, Y., He, Y., Qi, L., Jaddoe, V.W., Feskens, E.J., Yang, X., Ma, G., and Hu, F.B. (2010). Exposure to the Chinese famine in early life and the risk of hyperglycemia and type 2 diabetes in adulthood. Diabetes 59, 2400-2406. Fady, P.E., Dong, A., Gladyshev, V.N., et al. (2020). N6-adenosine methylation of ribosomal RNA affects lipid oxidation and stress resistance. Sci Adv 6, eaaz4370. phenomena to molecular mechanisms. Curr Opin Neurobiol 59, 189-206. Lim, J.P., and Brunet, A. (2013). Bridging the transgenerational gap with epigenetic memory. Trends Genet 29, 176-186. Liu, K., Santos, D.A., Hussmann, J.A., Wang, Y., Sutter, B.M., Weissman, J.S., and Tu, B.P. (2021). Regulation of translation by methylation multiplicity of $18 \mathrm{~S}$ rRNA. Cell Rep 34, 108825. RNA modification status at single nucleotide resolution in mRNA and long noncoding RNA. RNA $19,1848-1856$.

Love, M.I., Huber, W., and Anders, S. (2014). Moderated estimation of fold change and dispersion for RNA-seq data with DESeq2. Genome Biol 15, 550. Lumey, L.H., Stein, A.D., Kahn, H.S., and Romijn, J.A. (2009). Lipid profiles in middle-aged men and women after famine exposure during gestation: the Dutch Hunger Winter Families Study. Am J Clin Nutr 89, 1737-1743.

Mann, M.B., and Smith, H.O. (1977). Specificity of Hpa II and Hae III DNA methylases. Nucleic Acids Res 4, 4211-4221.

Martin, M. (2011). Cutadapt removes adapter sequences from high-throughput sequencing reads. EMBnet.journal 17.

Mello, C.C., Kramer, J.M., Stinchcomb, D., and Ambros, V. (1991). Efficient gene transfer in C.elegans: extrachromosomal maintenance and integration of transforming sequences. The EMBO journal 10, 3959-3970.

Migicovsky, Z., Yao, Y., and Kovalchuk, I. (2014). Transgenerational phenotypic and epigenetic changes in response to heat stress in Arabidopsis thaliana. Plant Signal Behav 9, e27971. Morana, A., Stiuso, P., Colonna, G., Lamberti, M., Carteni, M., and De Rosa, M. (2002). Stabilization of S-adenosyl-L-methionine promoted by trehalose. Biochim Biophys Acta 1573, 105-108.

Nikolskaya, II, Lopatina, N.G., and Debov, S.S. (1981). On heterogeneity of DNA methylases from Escherichia coli SK cells. Mol Cell Biochem 35, 3-10.

O'Connor, M., Thomas, C.L., Zimmermann, R.A., and Dahlberg, A.E. (1997). Decoding fidelity at the ribosomal $A$ and $P$ sites: influence of mutations in three different regions of the decoding domain in 16S rRNA. Nucleic Acids Res 25, 1185-1193.

1180 Painter, R.C., Osmond, C., Gluckman, P., Hanson, M., Phillips, D.I., and Roseboom, T.J. (2008).

1181 Transgenerational effects of prenatal exposure to the Dutch famine on neonatal adiposity and 1182 health in later life. BJOG 115, 1243-1249.

1183 Parks, L.W., and Schlenk, F. (1958). The stability and hydrolysis of S-adenosylmethionine; isolation of S-ribosylmethionine. The Journal of biological chemistry 230, 295-305. 
Pembrey, M.E., Bygren, L.O., Kaati, G., Edvinsson, S., Northstone, K., Sjostrom, M., and Golding, J. (2006). Sex-specific, male-line transgenerational responses in humans. European journal of human genetics : EJHG 14, 159-166.

Polikanov, Y.S., Melnikov, S.V., Soll, D., and Steitz, T.A. (2015). Structural insights into the role of rRNA modifications in protein synthesis and ribosome assembly. Nature structural \& molecular biology 22, 342-344.

Rechavi, O., Houri-Ze'evi, L., Anava, S., Goh, W.S., Kerk, S.Y., Hannon, G.J., and Hobert, O. (2014). Starvation-Induced Transgenerational Inheritance of Small RNAs in C. elegans. Cell 158, 277-287.

Schosserer, M., Minois, N., Angerer, T.B., Amring, M., Dellago, H., Harreither, E., Calle-Perez, A., Pircher, A., Gerstl, M.P., Pfeifenberger, S., et al. (2015). Methylation of ribosomal RNA by NSUN5 is a conserved mechanism modulating organismal lifespan. Nat Commun 6, 6158. Schott, D., Yanai, I., and Hunter, C.P. (2014). Natural RNA interference directs a heritable response to the environment. Sci Rep 4, 7387.

Seong, K.H., Li, D., Shimizu, H., Nakamura, R., and Ishii, S. (2011). Inheritance of stress-induced, ATF-2-dependent epigenetic change. Cell 145, 1049-1061.

Sergiev, P.V., Aleksashin, N.A., Chugunova, A.A., Polikanov, Y.S., and Dontsova, O.A. (2018). Structural and evolutionary insights into ribosomal RNA methylation. Nat Chem Biol 14, $226-$ 235.

Shen, H., Stoute, J., and Liu, K.F. (2020). Structural and catalytic roles of the human 18S rRNA methyltransferases DIMT1 in ribosome assembly and translation. The Journal of biological chemistry 295, 12058-12070.

Sloan, K.E., Warda, A.S., Sharma, S., Entian, K.D., Lafontaine, D.L.J., and Bohnsack, M.T. (2017). Tuning the ribosome: The influence of rRNA modification on eukaryotic ribosome biogenesis and function. RNA Biol 14, 1138-1152.

Su, D., Chan, C.T., Gu, C., Lim, K.S., Chionh, Y.H., McBee, M.E., Russell, B.S., Babu, I.R., Begley, T.J., and Dedon, P.C. (2014). Quantitative analysis of ribonucleoside modifications in tRNA by HPLC-coupled mass spectrometry. Nat Protoc 9, 828-841.

Subramanian, A., Tamayo, P., Mootha, V.K., Mukherjee, S., Ebert, B.L., Gillette, M.A., Paulovich, A., Pomeroy, S.L., Golub, T.R., Lander, E.S., et al. (2005). Gene set enrichment analysis: a knowledge-based approach for interpreting genome-wide expression profiles. Proceedings of the National Academy of Sciences of the United States of America 102, 15545-15550.

Suvorov, A.N., van Gemen, B., and van Knippenberg, P.H. (1988). Increased kasugamycin sensitivity in Escherichia coli caused by the presence of an inducible erythromycin resistance (erm) gene of Streptococcus pyogenes. Mol Gen Genet 215, 152-155.

Timmons, L., Court, D.L., and Fire, A. (2001). Ingestion of bacterially expressed dsRNAs can produce specific and potent genetic interference in Caenorhabditis elegans. Gene 263, 103-112. van Buul, C.P., Visser, W., and van Knippenberg, P.H. (1984). Increased translational fidelity caused by the antibiotic kasugamycin and ribosomal ambiguity in mutants harbouring the ksgA gene. FEBS Lett 177, 119-124.

Wang, R., Zheng, W., Yu, H., Deng, H., and Luo, M. (2011). Labeling substrates of protein arginine methyltransferase with engineered enzymes and matched S-adenosyl-L-methionine analogues. J Am Chem Soc 133, 7648-7651. 
1228 Webster, A.K., Jordan, J.M., Hibshman, J.D., Chitrakar, R., and Baugh, L.R. (2018).

1229 Transgenerational Effects of Extended Dauer Diapause on Starvation Survival and Gene

1230 Expression Plasticity in Caenorhabditis elegans. Genetics 210, 263-274.

1231 White, J., Li, Z., Sardana, R., Bujnicki, J.M., Marcotte, E.M., and Johnson, A.W. (2008). Bud23

1232 methylates G1575 of 18S rRNA and is required for efficient nuclear export of pre-40S subunits.

1233 Mol Cell Biol 28, 3151-3161.

1234 Zhu, C., Yan, Q., Weng, C., Hou, X., Mao, H., Liu, D., Feng, X., and Guang, S. (2018). Erroneous

1235 ribosomal RNAs promote the generation of antisense ribosomal siRNA. Proceedings of the

1236 National Academy of Sciences of the United States of America 115, 10082-10087.

1237 Zorbas, C., Nicolas, E., Wacheul, L., Huvelle, E., Heurgue-Hamard, V., and Lafontaine, D.L.

1238 (2015). The human 18S rRNA base methyltransferases DIMT1L and WBSCR22-TRMT112 but not rRNA modification are required for ribosome biogenesis. Mol Biol Cell 26, 2080-2095. 


\section{P0 fertility}

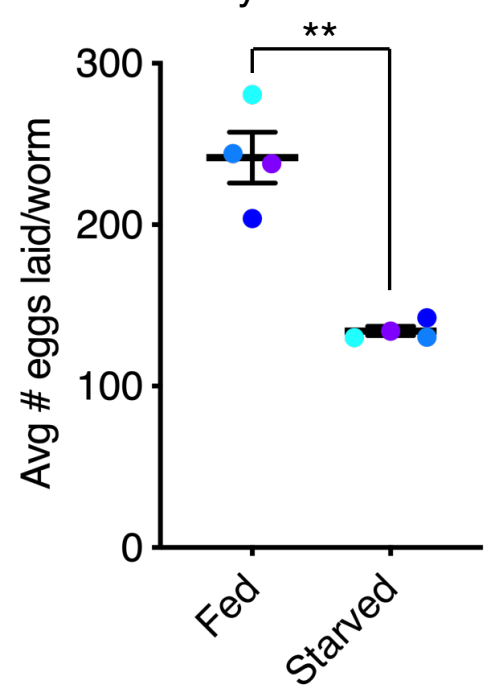

D

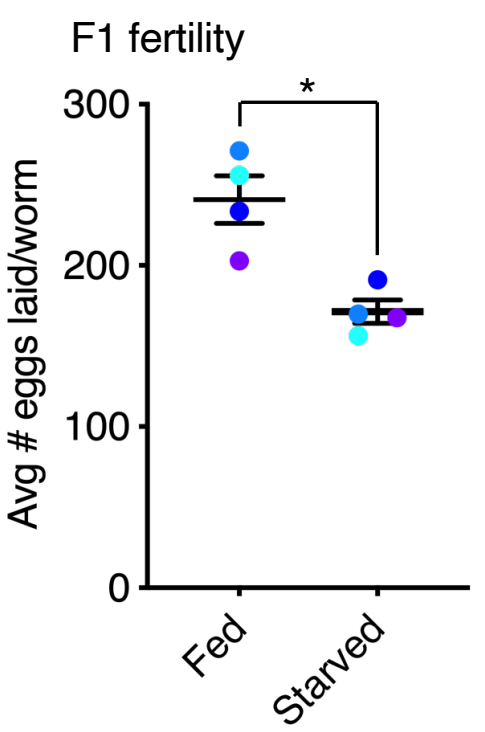

P0 heat stress

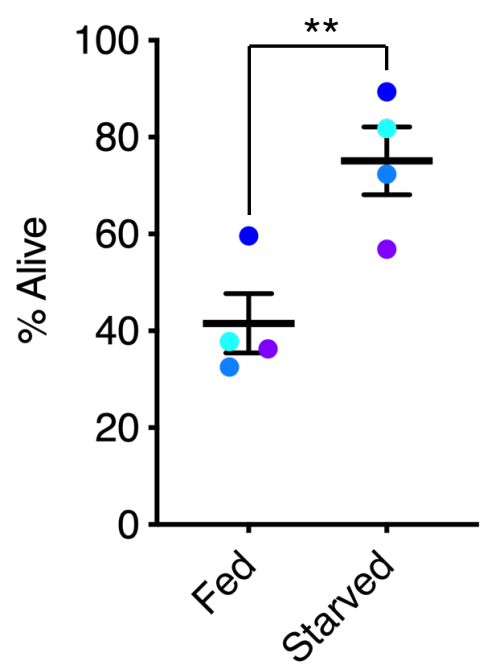

$\mathbf{E}$

F1 heat stress

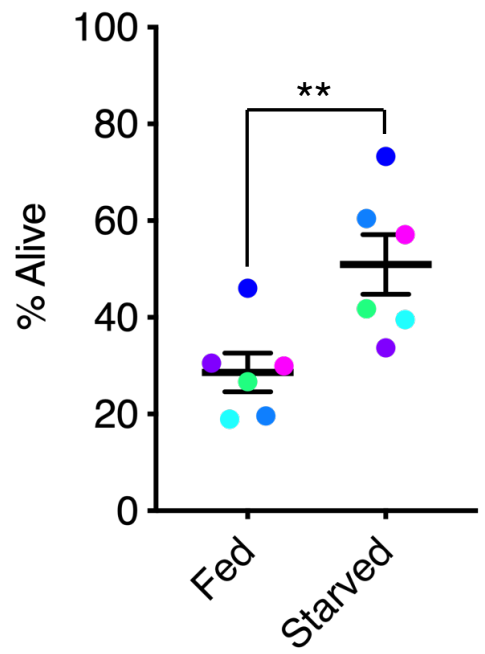

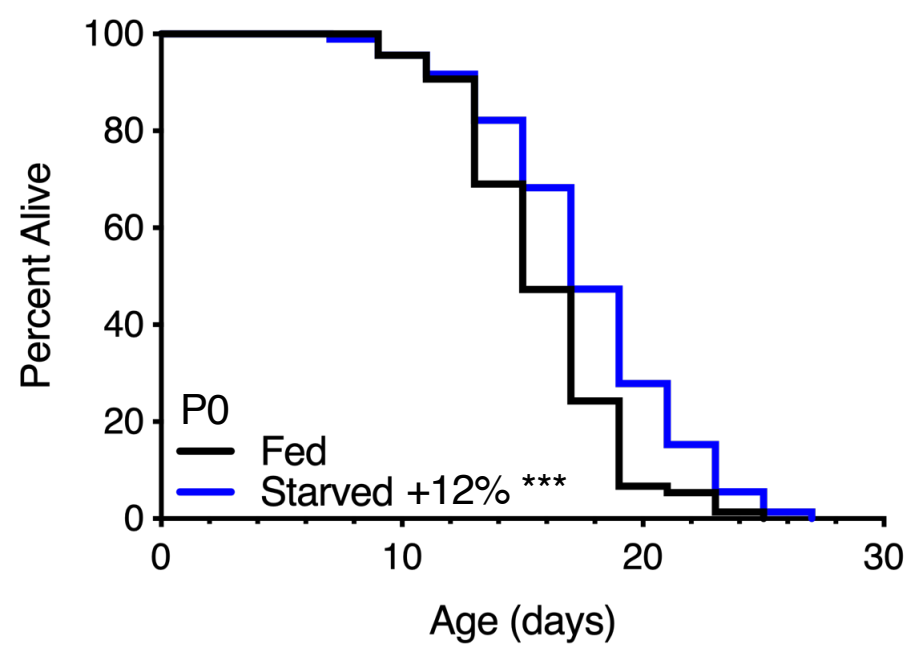

$\mathbf{F}$

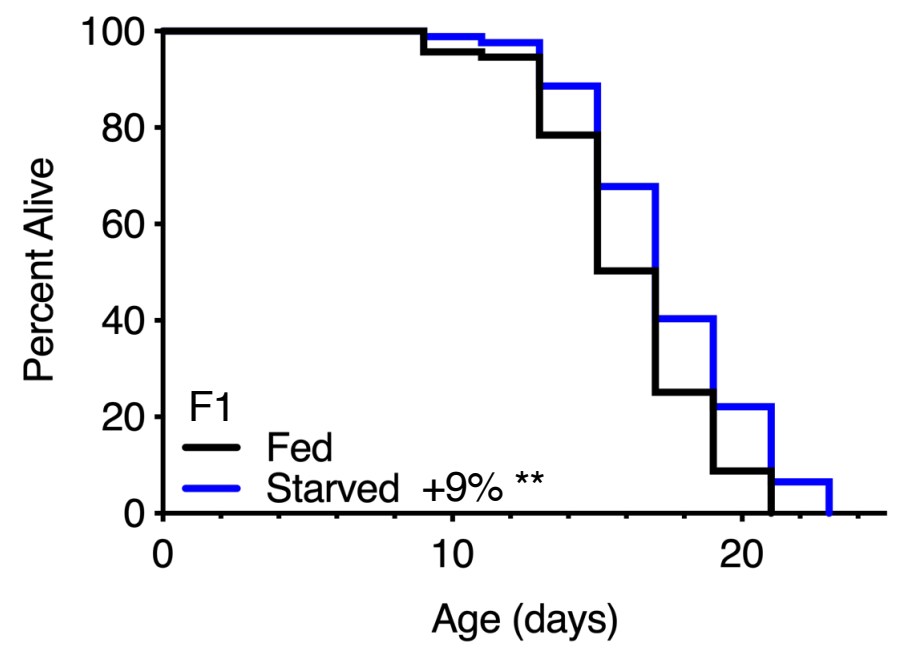

Fig. 1 Parental starvation causes intergenerational hormesis in descendants 
A

C
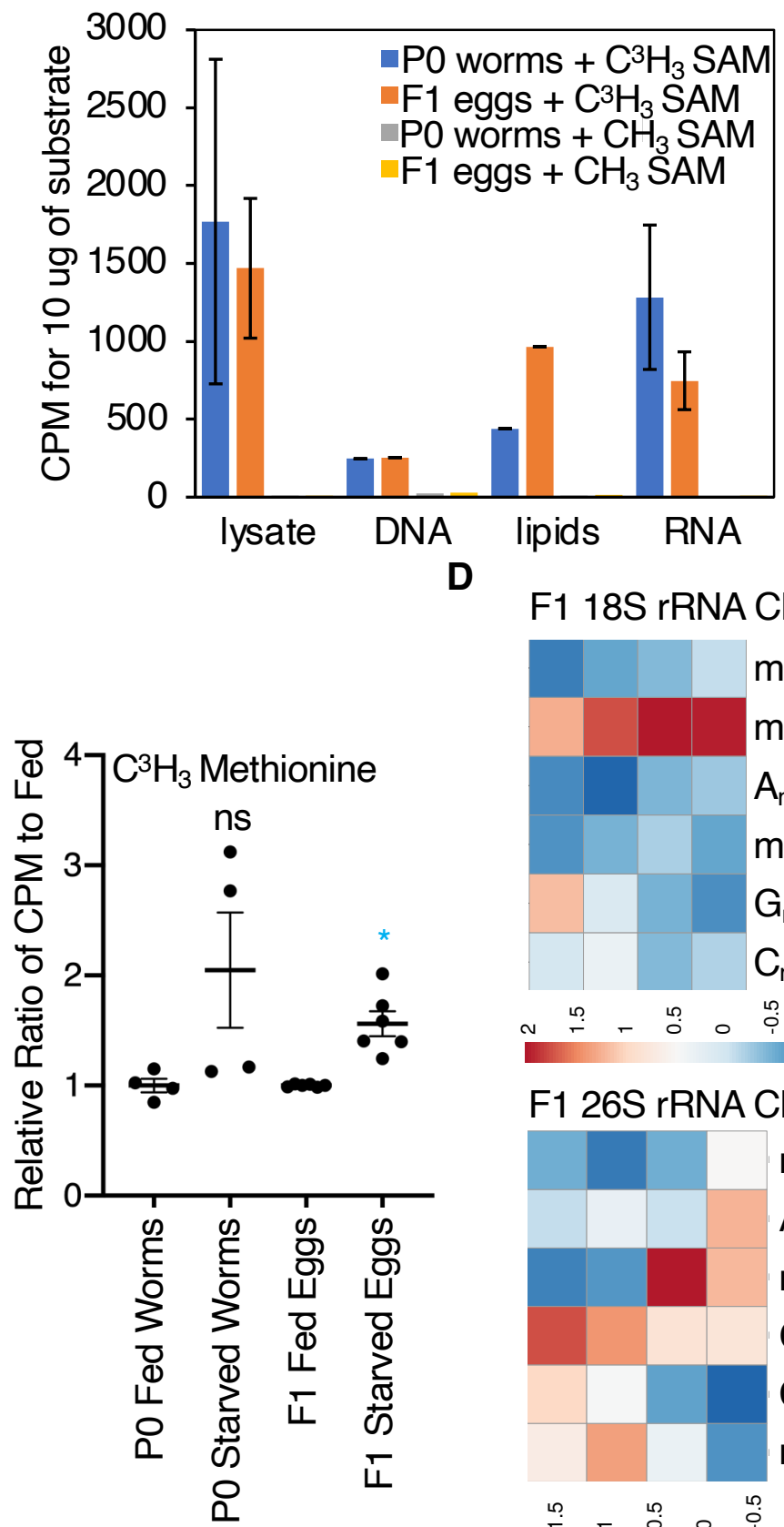

B D

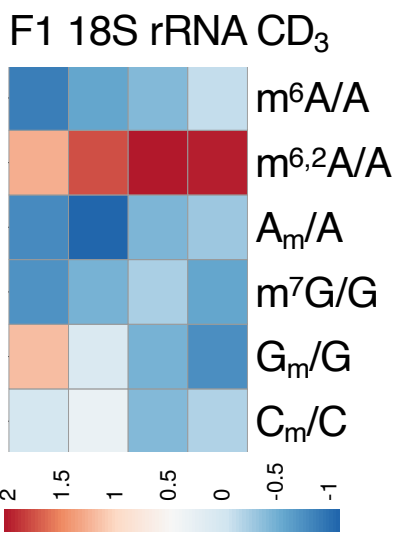

F1 26S rRNA $\mathrm{CD}_{3}$

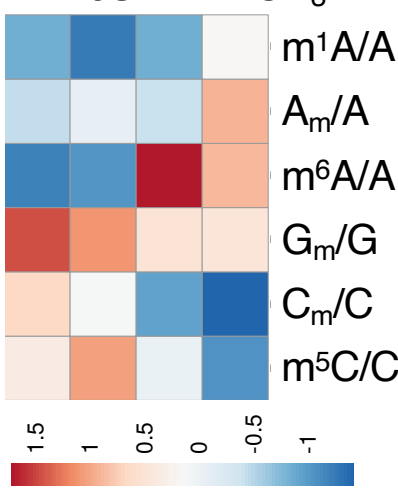

\section{Starved}

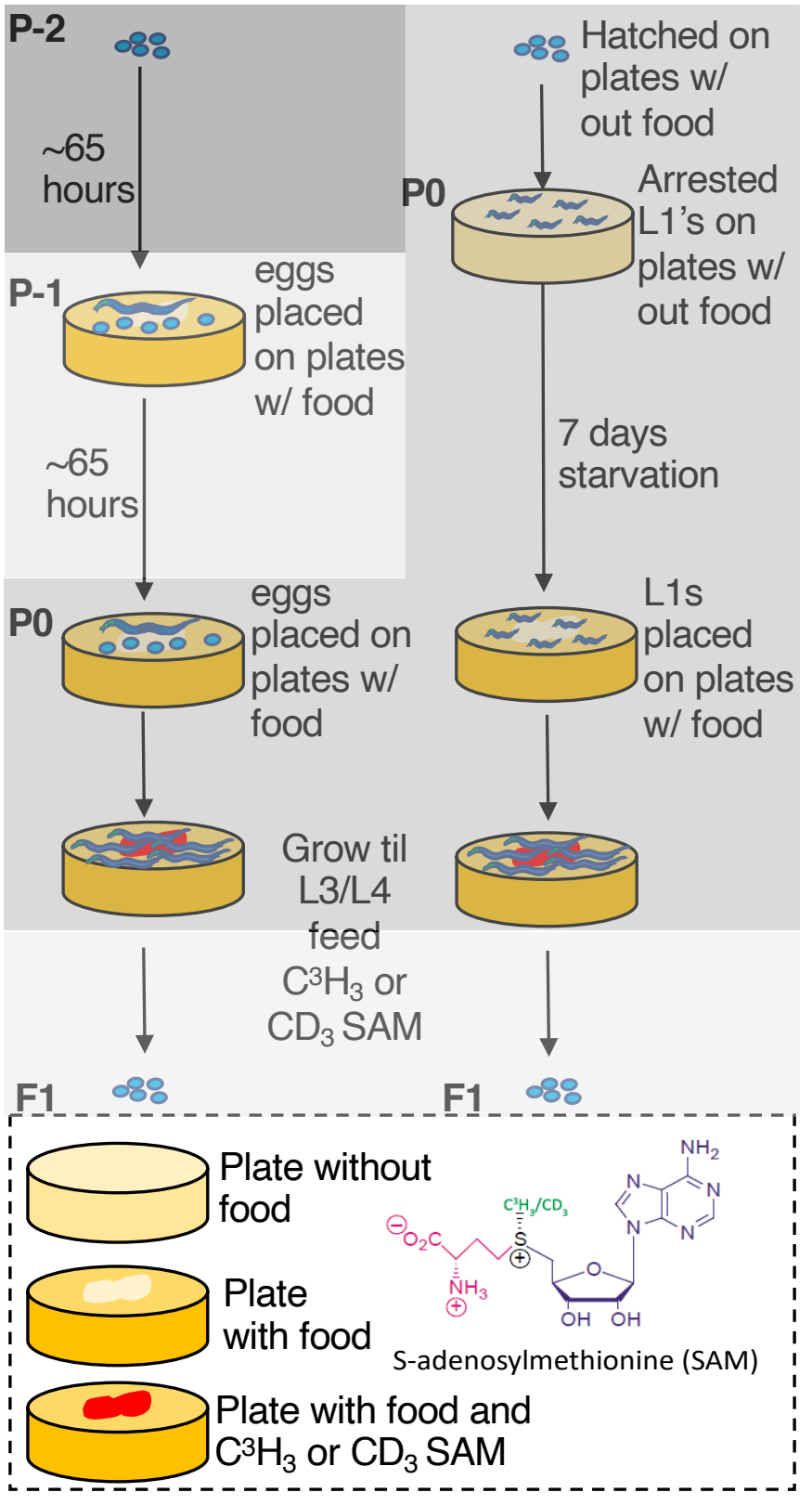




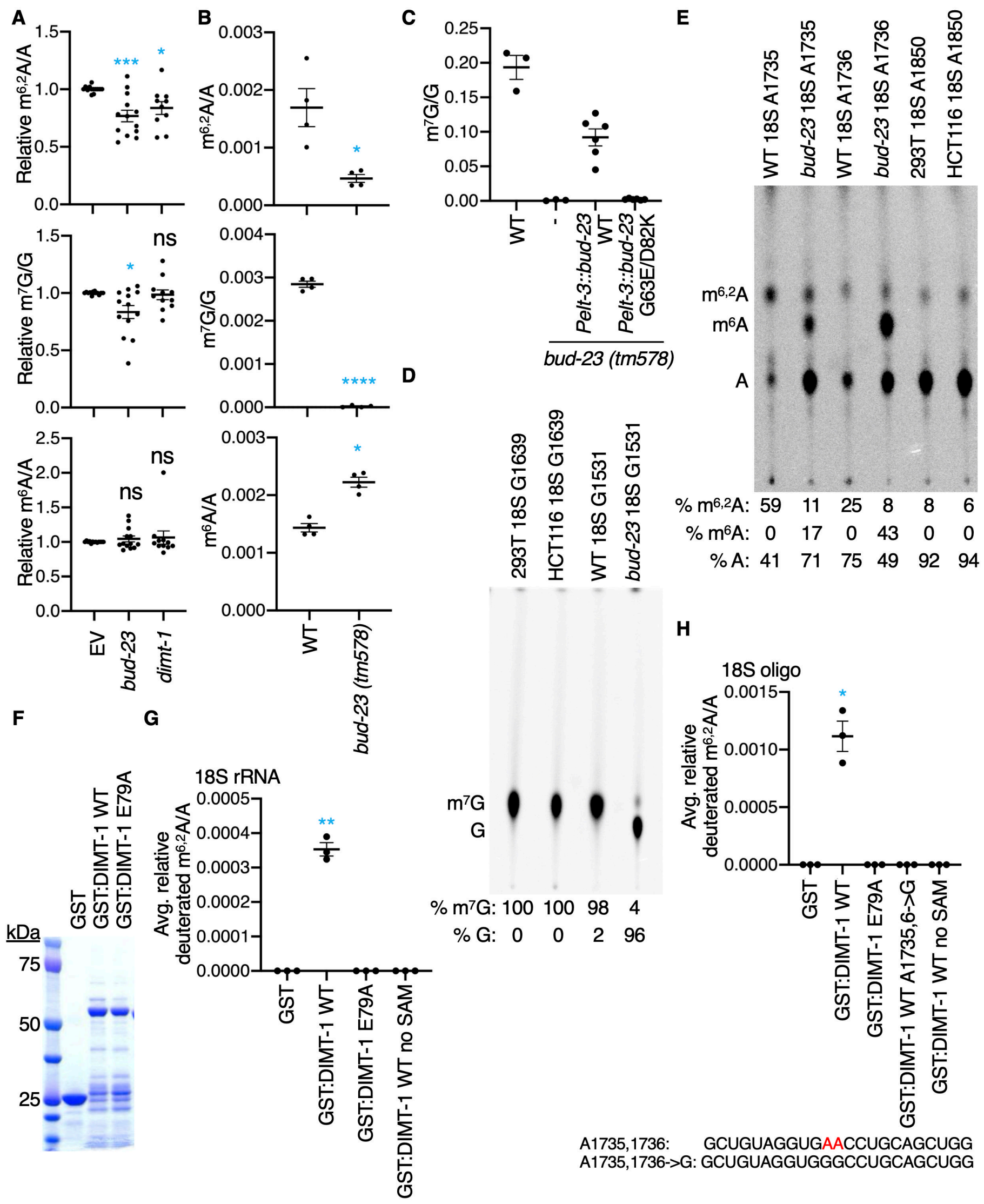

Fig. 3 DIMT-1 and BUD-23 are $m^{6,2} A$ and putative $m^{7} G$ 18S rRNA methyltransferases 
A

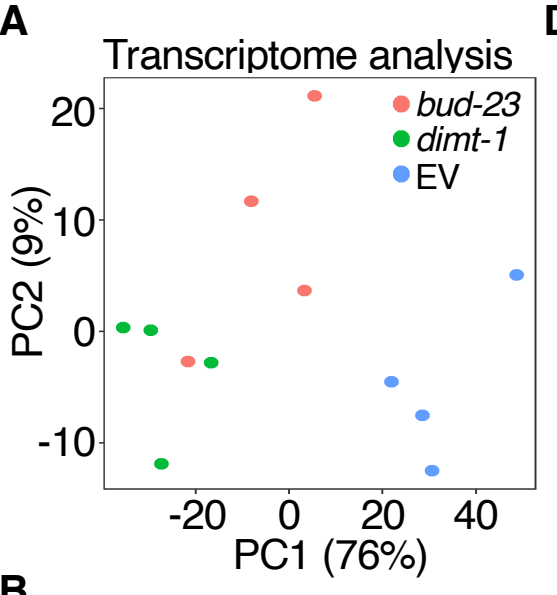

B

$\mathrm{KD}$ up regulated genes

bud-23 KD

(1319)

dimt-1 KD

(2802)

$95 \quad 1224$

1578

$\mathrm{KD}$ down regulated genes

bud-23 KD

(882)

dimt-1 KD

(2719)

$\begin{array}{lll}151 & 731 & 1988\end{array}$

C

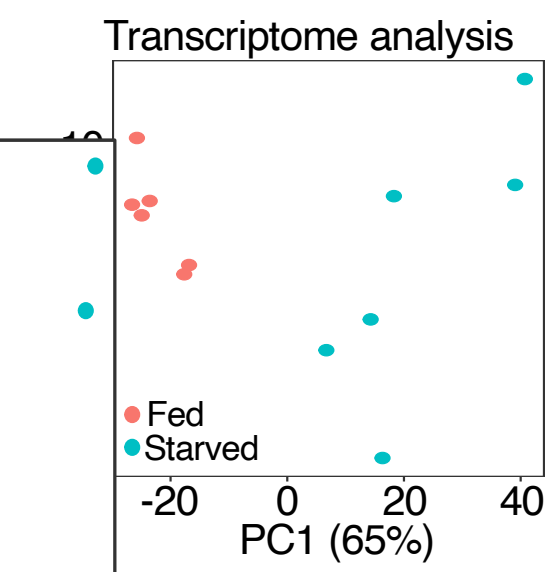

KD down regulated genes vs starved up regulated

bud-23 KD

(882)

dimt-1 KD

(2719)

195

Starved

(1648)

$\mathbf{F}$

Translation efficiency 的

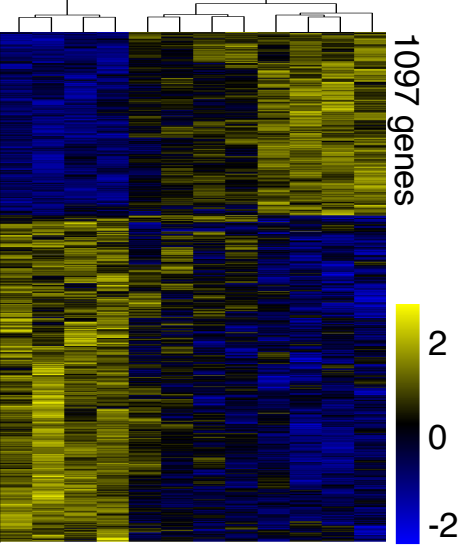

H

Translation efficiency

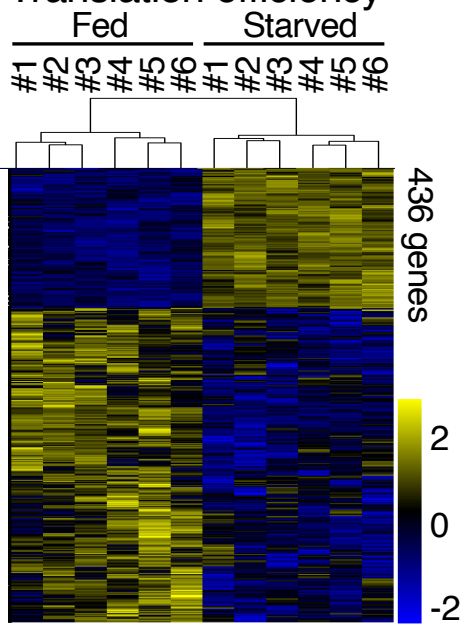

\section{I}

E

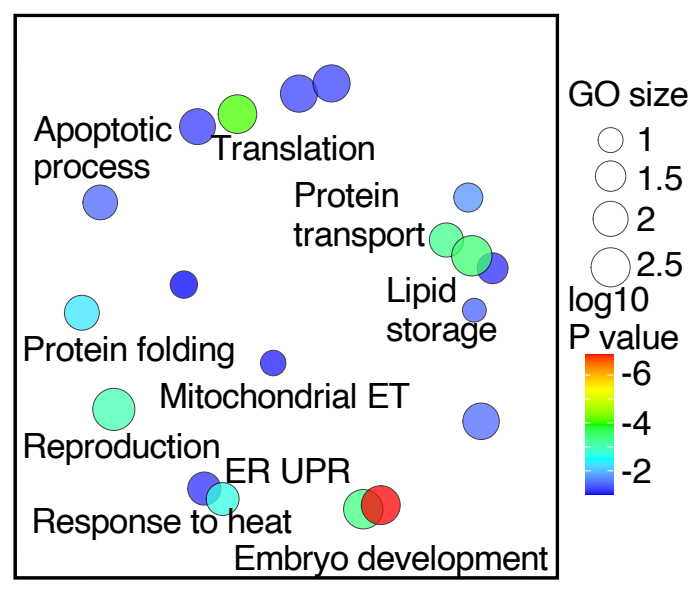

GO Term TE dimt-1 KD

$P$ value

Nematode larval development

Reproduction

$1.20 \mathrm{E}-21$

Embryo development

$7.58 \mathrm{E}-20$

Determination of adult lifespan

3.82E-18

Apoptotic process

$1.79 \mathrm{E}-9$

Cytosolic small ribosomal subunit 2.09E-8

Growth

8.42E-7

Ribosome

$1.42 \mathrm{E}-6$

Translation

2.44E-5

Endocytosis

$1.05 \mathrm{E}-4$

Neg. reg. of trans. from RNAPII 5.55E-4

Pos. reg. of trans. from RNAPII 1.23E-3

Response to heat

$1.75 \mathrm{E}-3$

$P$ granule

Response to starvation

$5.95 \mathrm{E}-3$

Proteolysis

7.92E-3

Cell death

$8.26 \mathrm{E}-3$

Translation elongation

$1.34 \mathrm{E}-2$

$1.70 \mathrm{E}-2$

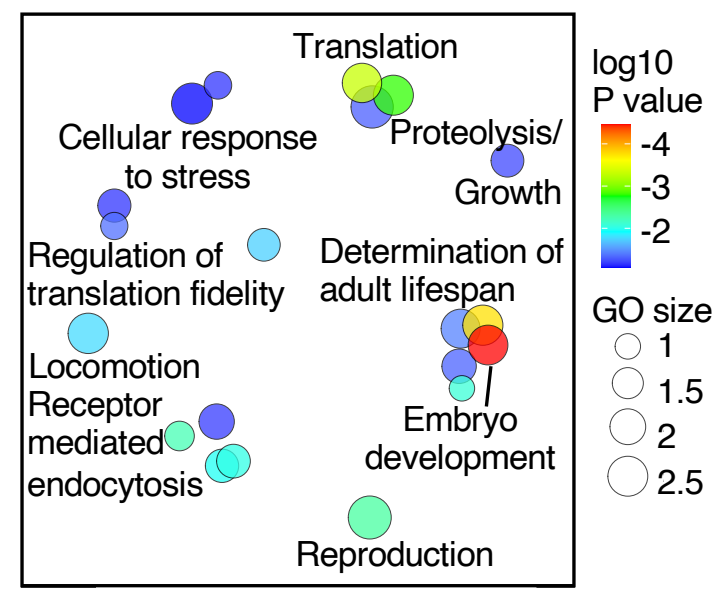

Fig. 4 Altered translation of genes involved in development, translation, longevity, and the stress response in response to bud-23 and dimt-1 KD and parental starvation 
A

P0 fertility

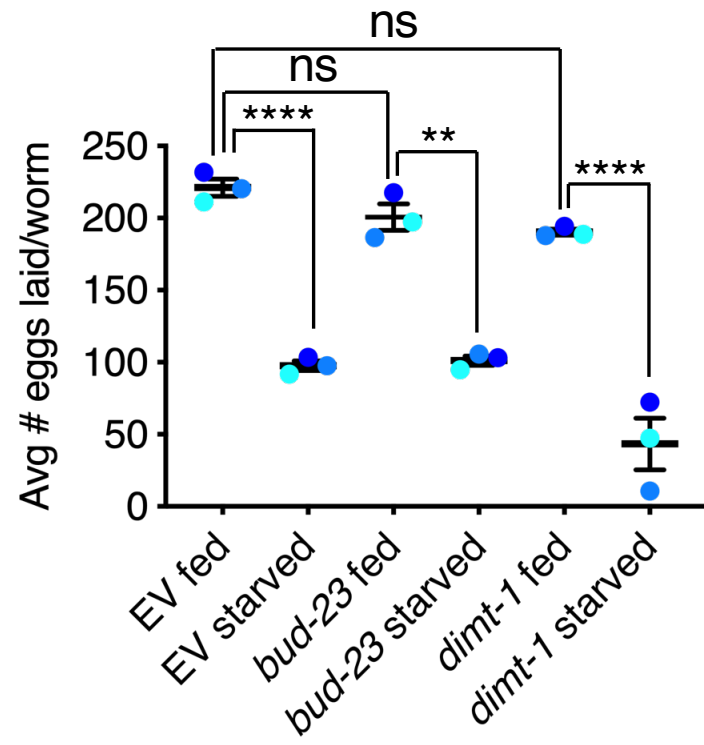

C

F1 fertility

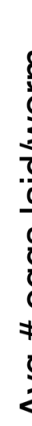

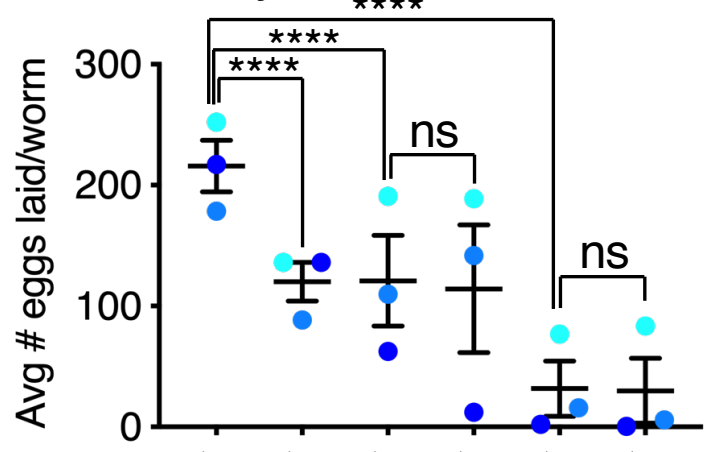

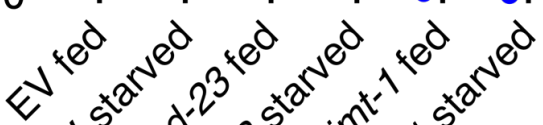

E

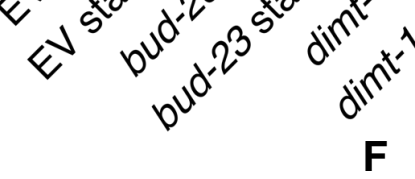

B

P0 heat stress

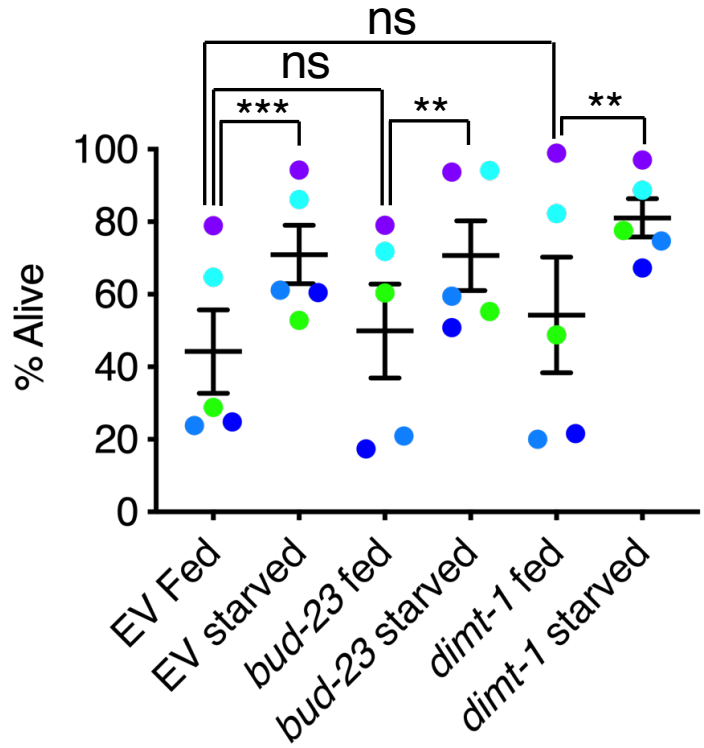

要

P0 fertility
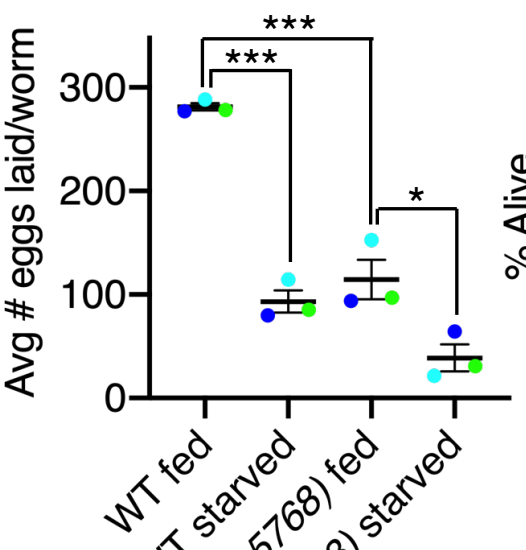

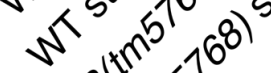

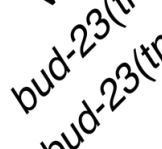

$\mathbf{F}$
F1 heat stress

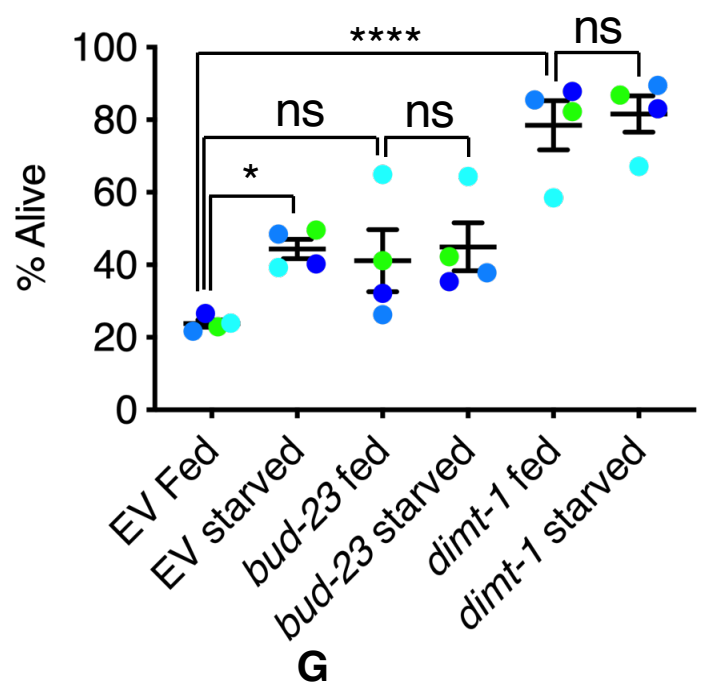

F1 fertility
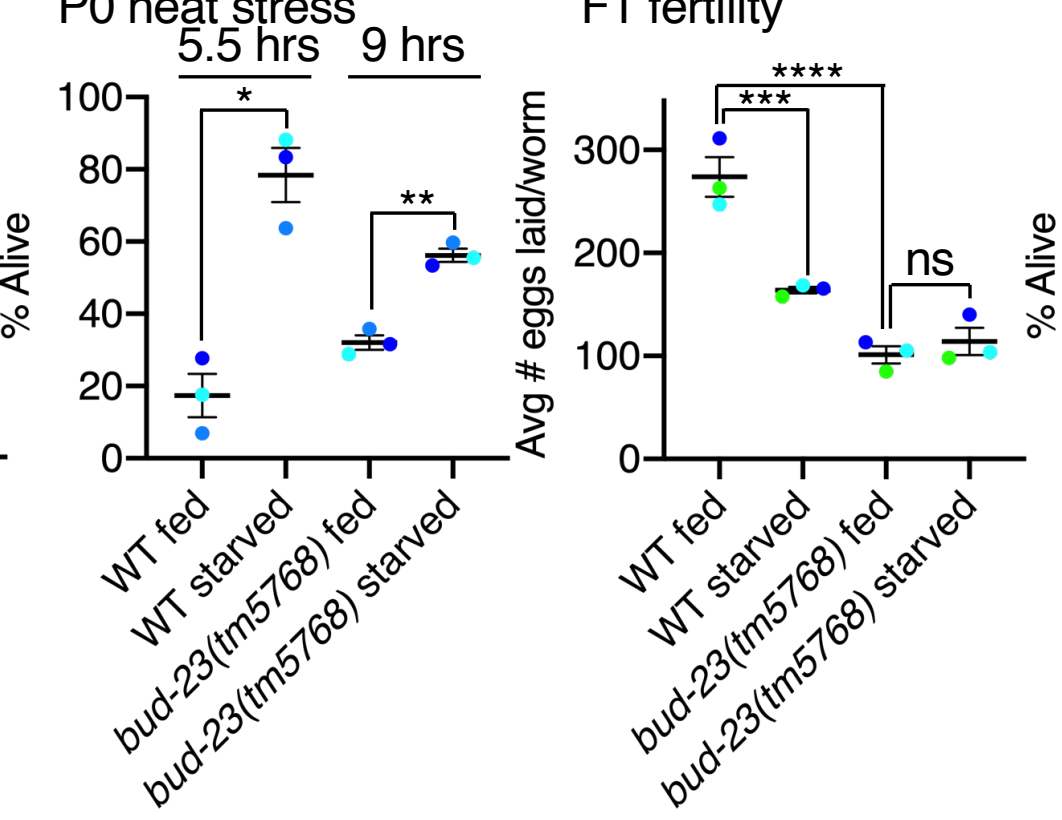

H

F1 heat stress

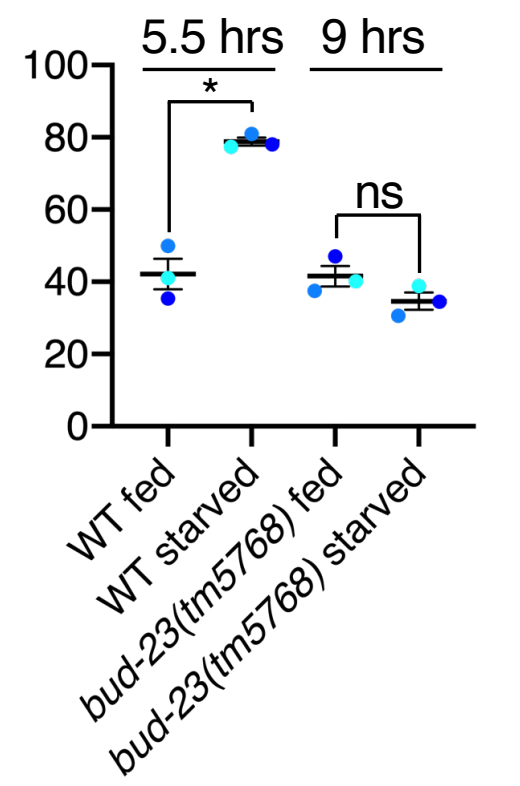

Fig. 5 dimt-1 and bud-23 are required for intergenerational hormesis 